\title{
Second-order integrable Lagrangians and WDVV equations
}

\author{
E. V. Ferapontov ${ }^{1,2}\left(\right.$ i) $\cdot$ M. V. Pavlov ${ }^{3} \cdot$ Lingling $X e^{3}$
}

Received: 10 October 2020 / Revised: 29 March 2021 / Accepted: 12 April 2021 /

Published online: 26 April 2021

(c) The Author(s) 2021

\section{Abstract}

We investigate the integrability of Euler-Lagrange equations associated with $2 \mathrm{D}$ second-order Lagrangians of the form

$$
\int f\left(u_{x x}, u_{x y}, u_{y y}\right) \mathrm{d} x \mathrm{~d} y .
$$

By deriving integrability conditions for the Lagrangian density $f$, examples of integrable Lagrangians expressible via elementary functions, Jacobi theta functions and dilogarithms are constructed. A link of second-order integrable Lagrangians to WDVV equations is established. Generalisations to 3D second-order integrable Lagrangians are also discussed.

Keywords Second-order Lagrangians - Systems of hydrodynamic type - Integrability (diagonalisability) conditions · Jacobi theta functions · Chazy equation, WDVV equations

Mathematics Subject Classification 35Q51 $\cdot 37 \mathrm{~K} 05 \cdot 37 \mathrm{~K} 10 \cdot 37 \mathrm{~K} 20 \cdot 53 \mathrm{D} 45$

Dedicated to the memory of Boris Anatolievich Dubrovin.

$\bowtie \quad$ E. V. Ferapontov

E.V.Ferapontov@lboro.ac.uk

M. V. Pavlov

mpavlov@itp.ac.ru

Lingling Xue

xuelingling@nbu.edu.cn

1 Department of Mathematical Sciences, Loughborough University, Leicestershire, Loughborough LE11 3TU, UK

2 Institute of Mathematics, Ufa Federal Research Centre, Russian Academy of Sciences, 112 Chernyshevsky Street, Ufa, Russian Federation 450008

3 Department of Applied Mathematics, Ningbo University, Ningbo 315211, People's Republic of China 


\section{Contents}

1 Introduction and summary of the main results . . . . . . . . . . . . . . . . . . . 2

2 Integrable Lagrangians in $2 \mathrm{D} \ldots \ldots \ldots \ldots \ldots \ldots$

2.1 Hydrodynamic form of Euler-Lagrange equations . . . . . . . . . . . . . . . . 4

2.2 Integrability conditions . . . . . . . . . . . . . . . . . . . . 6

2.3 Equivalence group in $2 \mathrm{D} \ldots \ldots \ldots \ldots \ldots \ldots \ldots$

2.4 Integrability conditions via generalised Rankin-Cohen brackets . . . . . . . . . . . . . . . 9

2.5 Integrable Lagrangians and classical Newtonian equations . . . . . . . . . . . . . . . . . . . . . . . . . . . . . . .

2.6 Integrable Lagrangians and WDVV equations . . . . . . . . . . . . . . . . . . . . . . . . . . . . . . . . . . . . . . .



2.7.1 Integrable Lagrangian densities of the form $f=g\left(u_{x x}, u_{y y}\right) \ldots \ldots \ldots 14$

2.7.2 Lagrangian densities for linearisable Euler-Lagrange equations . . . . . . . . . . . . 14

2.7.3 Integrable Lagrangian densities of the form $f=e^{u_{x x}} g\left(u_{x y}, u_{y y}\right) \ldots \ldots \ldots$

2.7.4 Integrable Lagrangian densities polynomial in $e^{u_{x x}}$ and $e^{u_{y y}} \ldots \ldots$. . . . . . . . . 18

2.7.5 Integrable Lagrangian densities from WDVV prepotentials . . . . . . . . . . . . . . . 19

2.7.6 WDVV prepotentials from integrable Lagrangian densities . . . . . . . . . . . . . . . . . 22

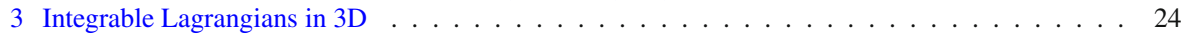

3.1 Integrability conditions . . . . . . . . . . . . . . . . . . . . . . . 24

3.2 Equivalence group in $3 \mathrm{D} \ldots \ldots \ldots \ldots \ldots \ldots$

3.3 Integrable Lagrangians associated with the dKP hierarchy . . . . . . . . . . . . . . . . . . . . . . . . . 26

3.4 2D densities as travelling wave reductions of 3D densities . . . . . . . . . . . . . . . . . . . . 27

4 Dispersive deformations of integrable Lagrangian densities . . . . . . . . . . . . . . . . . . . 28

5 Concluding remarks . . . . . . . . . . . . . . . . . . . . . . . 30

References................................. 31

\section{Introduction and summary of the main results}

We investigate second-order Lagrangians

$$
\int f\left(u_{x x}, u_{x y}, u_{y y}\right) \mathrm{d} x \mathrm{~d} y
$$

such that the corresponding Euler-Lagrange equations,

$$
\left(\frac{\partial f}{\partial u_{x x}}\right)_{x x}+\left(\frac{\partial f}{\partial u_{x y}}\right)_{x y}+\left(\frac{\partial f}{\partial u_{y y}}\right)_{y y}=0,
$$

are integrable (in the sense to be explained below). We work in the context of the formal calculus of variations and understand (1) as a formal action functional which generates Eq. (2) via the Euler-Lagrange operator applied to the Lagrangian density $f$. Examples of integrable Lagrangians (1) have appeared in the mathematical physics literature; thus, the Lagrangian density

$$
f=u_{x y}\left(u_{x x}^{2}-u_{y y}^{2}\right)+\alpha\left(u_{x x}^{2}-u_{y y}^{2}\right)+u_{x y}\left(\beta u_{x x}+\gamma u_{y y}\right)
$$

governs integrable geodesic flows on a 2-torus which possess a fourth-order integral polynomial in the momenta [3]. Similarly, the density

$$
f=u_{y y}^{2}+u_{x x}^{2} u_{y y}+u_{x x} u_{x y}^{2}+\frac{1}{4} u_{x x}^{4}
$$


governs integrable Newtonian equations possessing a fifth-order polynomial integral. In Sect. 2, we investigate the integrability aspects of 2D Lagrangians (1). Our main results can be summarised as follows.

- The Euler-Lagrange equation coming from Lagrangian (1) can be represented as a four-component Hamiltonian system of hydrodynamic type (Sect. 2.1). The requirement of its hydrodynamic integrability (which is equivalent to the vanishing of the corresponding Haantjes tensor) leads to an involutive system of third-order PDEs for the Lagrangian density $f$ (Sect. 2.2). Analysis of the integrability conditions reveals that integrable Lagrangians (1) locally depend on six arbitrary functions of one variable. Furthermore, the integrability conditions are themselves integrable — a standard phenomenon in the theory of integrable systems.

- The class of integrable Lagrangians (1) is invariant under the symplectic group $\operatorname{Sp}(4, \mathbb{R})$; under this action, the Lagrangian density $f$ transforms as a genus two Siegel modular form of weight -1 (Sect. 2.3). In particular, the integrability conditions can be represented via Sp-invariant operations known as generalised Rankin-Cohen (Eholzer-Ibukiyama) brackets (Sect. 2.4).

- Potentials $U(x, t)$ of classical Newtonian equations $\ddot{x}=-U_{x}$ that possess a fifth-order polynomial integral are governed by a Lagrangian (1) with density (4) (Sect. 2.5).

- Integrable Lagrangians (1) are related to WDVV prepotentials of the form

$$
F\left(t_{1}, t_{2}, t_{3}, t_{4}\right)=\frac{1}{2} t_{1}^{2} t_{4}+t_{1} t_{2} t_{3}+W\left(t_{2}, t_{3}, t_{4}\right)
$$

here, $W$ is a partial Legendre transform of the Lagrangian density $f$ (Sect. 2.6). This correspondence works both ways: using known solutions of WDVV equations, one can construct new integrable Lagrangians (1). Conversely, integrable Lagrangian densities $f$ give rise to WDVV prepotentials. Examples of this kind are given in Sects. 2.7.5 and 2.7.6.

- Further examples of integrable Lagrangians (1) expressible via elementary functions, Jacobi theta functions and dilogaritms are constructed in Sect. 2.7.

In Sect. 3, we investigate 3D second-order Lagrangians of the form

$$
\int f\left(u_{x x}, u_{x y}, u_{x t}, u_{y y}, u_{y t}, u_{t t}\right) \mathrm{d} x \mathrm{~d} y \mathrm{~d} t .
$$

Our results can be summarised as follows:

- Integrable Lagrangians in 3D are governed by a third-order PDE system for the Lagrangian density $f$ which comes from the requirement that all travelling wave reductions of a 3D Lagrangian to 2D are integrable in the sense of Sect. 2 (Sect. 3.1).

- The class of integrable Lagrangians (5) is invariant under the symplectic group $\operatorname{Sp}(6, \mathbb{R})$; the Lagrangian density $f$ transforms as a genus three Siegel modular form of weight -1 (Sect. 3.2). 
- Examples of integrable Lagrangians (5) are constructed in Sect. 3.3. These include the densities

$$
\begin{aligned}
& f=u_{y y}^{2}-u_{x x} u_{x t}+u_{x x}^{2} u_{y y}+u_{x x} u_{x y}^{2}+\frac{1}{4} u_{x x}^{4}, \\
& f=\left(u_{x y}-u_{t t}-u_{x x} u_{x t}+\frac{1}{3} u_{x x}^{3}\right)^{3 / 2}, \\
& f=u_{x t}^{-2}\left(u_{x t} u_{y t}-u_{x x} u_{x t}^{2}\right)^{3 / 2},
\end{aligned}
$$

coming from the theory of the dispersionless KP hierarchy (Sect. 3.3).

In Sect. 4, we discuss examples of integrable dispersive deformations of integrable Lagrangian densities (5). The general problem of constructing such deformations is largely open.

Our interest in second-order integrable Lagrangians stems from the study of 3D first-order Lagrangians

$$
\int f\left(u_{x}, u_{y}, u_{t}\right) \mathrm{d} x \mathrm{~d} y \mathrm{~d} t
$$

initiated in [23]. It was pointed out in [24] that the generic integrable Lagrangian density $f\left(u_{x}, u_{y}, u_{t}\right)$ is an automorphic function of its arguments. Let us emphasise an important difference between first-order and second-order Lagrangians in 2D: all first-order Lagrangian densities $f\left(u_{x}, u_{y}\right)$ lead to linearisable Euler-Lagrange equations which, therefore, are automatically integrable. On the contrary, for second-order Lagrangian densities $f\left(u_{x x}, u_{x y}, u_{y y}\right)$, the integrability conditions of the corresponding Euler-Lagrange equations are already highly non-trivial.

\section{Integrable Lagrangians in 2D}

In this section, we consider second-order integrable Lagrangians of type (1),

$$
\int f\left(u_{x x}, u_{x y}, u_{y y}\right) \mathrm{d} x \mathrm{~d} y .
$$

\subsection{Hydrodynamic form of Euler-Lagrange equations}

The Euler-Lagrange equation (2) is a fourth-order PDE for $u(x, y)$. Setting $a=u_{x x}$, $b=u_{x y}, c=u_{y y}$, we can rewrite (2) in the form

$$
b_{x}=a_{y}, \quad c_{x}=b_{y}, \quad\left(f_{a}\right)_{x x}+\left(f_{b}\right)_{x y}+\left(f_{c}\right)_{y y}=0 .
$$

Introducing the auxiliary variable $p$ via the relations

$$
p_{y}=-\left(f_{a}\right)_{x}, \quad p_{x}=\left(f_{b}\right)_{x}+\left(f_{c}\right)_{y},
$$


we can rewrite (6) as a first-order four-component conservative system

$$
a_{y}=b_{x}, b_{y}=c_{x}, \quad\left(f_{c}\right)_{y}=\left(p-f_{b}\right)_{x}, \quad p_{y}=-\left(f_{a}\right)_{x}
$$

or, in matrix form,

$$
\mathbf{R} w_{y}=\mathbf{S} w_{x}
$$

where $w=(a, b, c, p)^{T}$ and

$$
\mathbf{R}=\left(\begin{array}{cccc}
1 & 0 & 0 & 0 \\
0 & 1 & 0 & 0 \\
f_{a c} & f_{b c} & f_{c c} & 0 \\
0 & 0 & 0 & 1
\end{array}\right), \quad \mathbf{S}=\left(\begin{array}{cccc}
0 & 1 & 0 & 0 \\
0 & 0 & 1 & 0 \\
-f_{a b}-f_{b b}-f_{b c} & 1 \\
-f_{a a}-f_{a b}-f_{a c} & 0
\end{array}\right)
$$

Assuming $f_{c c} \neq 0$, we obtain a four-component system of hydrodynamic type,

$$
w_{y}=\mathbf{V}(w) w_{x}, \quad \mathbf{V}(w)=\mathbf{R}^{-1} \mathbf{S}
$$

Note that the restriction $f_{c c} \neq 0$ is only needed for the matrix $\mathbf{R}$ to be invertible and is not really important. One can always make a suitable linear change of the independent variables $x, y$ to transform the Euler-Lagrange equation into a hydrodynamic form.

Remark 1 System (7) can be put into a Hamiltonian form. For that purpose, we introduce the new dependent variables $(A, B, C)$ which are related to $(a, b, c)$ via partial Legendre transform,

$$
A=a, B=b, C=f_{c}, h=c f_{c}-f, h_{A}=-f_{a}, h_{B}=-f_{b}, h_{C}=c .
$$

In the new variables, system (7) takes the form $(P=p)$

$$
A_{y}=B_{x}, B_{y}=\left(h_{C}\right)_{x}, \quad C_{y}=\left(P+h_{B}\right)_{x}, \quad P_{y}=\left(h_{A}\right)_{x} \text {, }
$$

which is manifestly Hamiltonian:

$$
\left(\begin{array}{l}
A \\
B \\
C \\
P
\end{array}\right)_{y}=\left(\begin{array}{llll}
0 & 0 & 0 & 1 \\
0 & 0 & 1 & 0 \\
0 & 1 & 0 & 0 \\
1 & 0 & 0 & 0
\end{array}\right) \frac{\mathrm{d}}{\mathrm{d} x}\left(\begin{array}{l}
H_{A} \\
H_{B} \\
H_{C} \\
H_{P}
\end{array}\right)
$$

with the Hamiltonian density $H=h(A, B, C)+B P$. 


\subsection{Integrability conditions}

Since hydrodynamic-type system (7) is conservative, its integrability by the generalised hodograph method $[37,38]$ is equivalent to the diagonalisability of the matrix $\mathbf{V}(w)$ from (8). This is equivalent to the vanishing of the corresponding Haantjes tensor [25]. Recall that the Nijenhuis tensor of the matrix $\mathbf{V}(w)=\left(v_{j}^{i}(w)\right)$ is defined as

$$
N_{j k}^{i}=v_{j}^{s} \partial_{w^{s}} v_{k}^{i}-v_{k}^{s} \partial_{w^{s}} v_{j}^{i}-v_{s}^{i}\left(\partial_{w^{j}} v_{k}^{s}-\partial_{w^{k}} v_{j}^{s}\right)
$$

where we adopt the notation $w=\left(w^{1}, w^{2}, w^{3}, w^{4}\right)^{T}=(a, b, c, p)^{T}$. The Haantjes tensor is defined as

$$
H_{j k}^{i}=N_{s r}^{i} v_{j}^{s} v_{k}^{r}+N_{j k}^{s} v_{r}^{i} v_{s}^{r}-N_{j r}^{s} v_{s}^{i} v_{k}^{r}-N_{r k}^{s} v_{s}^{i} v_{j}^{r}
$$

It is easy to see that both tensors are skew-symmetric in the low indices. The requirement of vanishing of the Haantjes tensor leads to a system of PDEs (integrability conditions) for the Lagrangian density $f(a, b, c)$ which can be represented in a symmetric conservative form:

$$
\begin{gathered}
\left(f_{a b} f_{c c}-f_{a c} f_{b c}\right)_{a}=\left(f_{b c} f_{a a}-f_{a b} f_{a c}\right)_{c}, \\
\left(f_{a a} f_{c c}-f_{a c}^{2}\right)_{a}=\left(f_{a a} f_{b b}-f_{a b}^{2}\right)_{c}, \\
\left(f_{a a} f_{c c}-f_{a c}^{2}\right)_{c}=\left(f_{c c} f_{b b}-f_{b c}^{2}\right)_{a}, \\
\left(f_{b b} f_{c c}-f_{b c}^{2}\right)_{b}=2\left(f_{a b} f_{c c}-f_{a c} f_{b c}\right)_{c}, \\
\left(f_{b b} f_{a a}-f_{a b}^{2}\right)_{b}=2\left(f_{b c} f_{a a}-f_{a c} f_{a b}\right)_{a} .
\end{gathered}
$$

(Note that the stronger condition of vanishing of the Nijenhuis tensor leads to quadratic densities $f$ with linear Euler-Lagrange equations). Integrability conditions (10) are invariant under the discrete symmetries $a \leftrightarrow c$ and $b \rightarrow-b$. Indeed, under the interchange of $a$ and $c$ Eq. (10) 1 stays the same, while $(10)_{2},(10)_{3}$ and $(10)_{4},(10)_{5}$ get interchanged. Strictly speaking, the vanishing of the Haantjes tensor gives only the first four of relations (10); however, one can show that the fifth follows from the first four. We prefer to keep all of them for symmetry reasons.

Theorem 1 The system of integrability conditions (10) is in involution. The general 2D integrable Lagrangian density $f$ depends on six arbitrary functions of one variable.

Proof Let us introduce the new dependent variables

$$
s=\left(s_{1}, s_{2}, s_{3}, s_{4}, s_{5}, s_{6}\right)^{T}=\left(f_{a a}, f_{a b}, f_{a c}, f_{b b}, f_{b c}, f_{c c}\right)^{T},
$$


which satisfy the obvious consistency conditions such as

$$
\begin{gathered}
\left(s_{1}\right)_{b}=\left(s_{2}\right)_{a},\left(s_{1}\right)_{c}=\left(s_{3}\right)_{a},\left(s_{2}\right)_{b}=\left(s_{4}\right)_{a},\left(s_{2}\right)_{c}=\left(s_{3}\right)_{b}=\left(s_{5}\right)_{a}, \\
\left(s_{3}\right)_{c}=\left(s_{6}\right)_{a},\left(s_{4}\right)_{c}=\left(s_{5}\right)_{b},\left(s_{5}\right)_{c}=\left(s_{6}\right)_{b} .
\end{gathered}
$$

Taking these consistency conditions along with the integrability conditions (10), also rewritten in terms of $s$-variables, we obtain a system of twelve first-order quasilinear equations for $s_{i}(a, b, c)$ which can be represented in the form of two six-component systems of hydrodynamic type,

$$
s_{a}=\mathbf{P}(s) s_{c}, \quad s_{b}=\mathbf{Q}(s) s_{c},
$$

where $\mathbf{P}, \mathbf{Q}$ are the following $6 \times 6$ matrices:

$$
\begin{aligned}
& \mathbf{P}=\frac{1}{s_{6}}\left(\begin{array}{llllll}
2 s_{3}+s_{4} & -2 s_{2} & -s_{1} & s_{1} & 0 & 0 \\
2 s_{5} & 0 & -2 s_{2} & 0 & s_{1} & 0 \\
s_{6} & 0 & 0 & 0 & 0 & 0 \\
s_{6} & 2 s_{5} & -2 s_{3}-s_{4} & 0 & 0 & s_{1} \\
0 & s_{6} & 0 & 0 & 0 & 0 \\
0 & 0 & s_{6} & 0 & 0 & 0
\end{array}\right) \\
& \mathbf{Q}=\frac{1}{s_{6}}\left(\begin{array}{cccccc}
2 s_{5} & 0 & -2 s_{2} & 0 & s_{1} & 0 \\
s_{6} & 2 s_{5} & -2 s_{3}-s_{4} & 0 & 0 & s_{1} \\
0 & s_{6} & 0 & 0 & 0 & 0 \\
0 & 2 s_{6} & -2 s_{5} & 2 s_{5} & -2 s_{3}-s_{4} & 2 s_{2} \\
0 & 0 & 0 & s_{6} & 0 & 0 \\
0 & 0 & 0 & 0 & s_{6} & 0
\end{array}\right) \text {. }
\end{aligned}
$$

Equation (11) possess six conserved densities $s_{2}, s_{4}, s_{5}, s_{1} s_{5}-s_{2} s_{3}, s_{2} s_{6}-$ $s_{3} s_{5}, s_{1} s_{6}+s_{2} s_{5}-s_{3} s_{4}-s_{3}^{2}$ which satisfy the equations

$$
\begin{aligned}
& \left(s_{2}\right)_{a}=\left(s_{1}\right)_{b}, \quad\left(s_{4}\right)_{a}=\left(s_{2}\right)_{b}, \quad\left(s_{5}\right)_{a}=\left(s_{3}\right)_{b}, \\
& \left(s_{2}\right)_{c}=\left(s_{3}\right)_{b}, \quad\left(s_{4}\right)_{c}=\left(s_{5}\right)_{b}, \quad\left(s_{5}\right)_{c}=\left(s_{6}\right)_{b}, \\
& 2\left(s_{1} s_{5}-s_{2} s_{3}\right)_{a}=\left(s_{1} s_{4}-s_{2}^{2}\right)_{b}, \quad 2\left(s_{2} s_{6}-s_{3} s_{5}\right)_{a}=\left(s_{1} s_{6}-s_{3}^{2}\right)_{b}, \\
& 2\left(s_{1} s_{5}-s_{2} s_{3}\right)_{c}=\left(s_{1} s_{6}-s_{3}^{2}\right)_{b}, \quad 2\left(s_{2} s_{6}-s_{3} s_{5}\right)_{c}=\left(s_{4} s_{6}-s_{5}^{2}\right)_{b}, \\
& \left(s_{1} s_{6}+s_{2} s_{5}-s_{3} s_{4}-s_{3}^{2}\right)_{a}=\left(s_{1} s_{5}-s_{2} s_{3}\right)_{b}, \\
& \left(s_{1} s_{6}+s_{2} s_{5}-s_{3} s_{4}-s_{3}^{2}\right)_{c}=\left(s_{2} s_{6}-s_{3} s_{5}\right)_{b} .
\end{aligned}
$$

Direct calculation shows that systems (11) commute, that is, $s_{a b}=s_{b a}$. Thus, Eq. (11) are in involution, and their general common solution depends on six arbitrary 
functions of one variable, namely the Cauchy data $s_{i}(0,0, c)$. This finishes the proof.

Remark 2 Relations (10) and (12) imply that there exists a potential $\rho$ such that

$$
\begin{aligned}
& \rho_{a a}=f_{a a} f_{b b}-f_{a b}^{2}, \quad \rho_{a c}=f_{a a} f_{c c}-f_{a c}^{2}, \quad \rho_{c c}=f_{c c} f_{b b}-f_{b c}^{2}, \\
& \rho_{a b}=2\left(f_{b c} f_{a a}-f_{a c} f_{a b}\right), \quad \rho_{b c}=2\left(f_{a b} f_{c c}-f_{a c} f_{b c}\right), \\
& \rho_{b b}=2\left(f_{a b} f_{b c}-f_{a c} f_{b b}+f_{a a} f_{c c}-f_{a c}^{2}\right) .
\end{aligned}
$$

Remark 3 System (10)

possesses a Lax pair

$$
\psi_{a}=\lambda \mathbf{K} \psi, \quad \psi_{b}=\lambda \mathbf{L} \psi, \quad \psi_{c}=\lambda \mathbf{M} \psi
$$

where $\lambda$ is a spectral parameter and the $4 \times 4$ matrices $\mathbf{K}, \mathbf{L}, \mathbf{M}$ are defined as

$$
\begin{aligned}
& \mathbf{K}=\left(\begin{array}{cccc}
0 & f_{a c} & 0 & 1 \\
-f_{a a} & -f_{a b} & 0 & 0 \\
-\frac{1}{2} \rho_{a b} & -\rho_{a c} & -f_{a b} & f_{a c} \\
-\rho_{a a} & -\frac{1}{2} \rho_{a b} & -f_{a a} & 0
\end{array}\right), \quad \mathbf{L}=\left(\begin{array}{cccc}
0 & f_{b c} & 1 & 0 \\
-f_{a b} & -f_{b b} & 0 & 1 \\
-\frac{1}{2} \rho_{b b} & -\rho_{b c} & -f_{b b} & f_{b c} \\
-\rho_{a b} & -\frac{1}{2} \rho_{b b} & -f_{a b} & 0
\end{array}\right), \\
& \mathbf{M}=\left(\begin{array}{cccc}
0 & f_{c c} & 0 & 0 \\
-f_{a c} & -f_{b c} & 1 & 0 \\
-\frac{1}{2} \rho_{b c} & -\rho_{c c} & -f_{b c} & f_{c c} \\
-\rho_{a c} & -\frac{1}{2} \rho_{b c} & -f_{a c} & 0
\end{array}\right)
\end{aligned}
$$

here the potential $\rho$ is defined by relations (13).

Remark 4 We have verified that both commuting six-component systems (11) satisfy the following properties:

- They are linearly degenerate, that is, Lie derivatives of the eigenvalues of matrices $\mathbf{P}, \mathbf{Q}$ along the corresponding eigenvectors are zero, see, for example, [29]. The condition of linear degeneracy can be efficiently verified using a criterion from [19].

- They are non-diagonalisable (their Haantjes tensor does not vanish).

This suggests that integrable Lagrangian densities (1) are related to the associativity (WDVV) equations where analogous systems were obtained in [22], see also [33,34] for related results. Such a link indeed exists and is discussed in Sect. 2.6.

\subsection{Equivalence group in 2D}

Let $\mathbf{U}$ be the $2 \times 2$ Hessian matrix of the function $u(x, y)$. Integrable Lagrangians of type (1) are invariant under $\operatorname{Sp}(4, \mathbb{R})$-symmetry

$$
\mathbf{U} \rightarrow(\mathbf{A} \mathbf{U}+\mathbf{B})(\mathbf{C} \mathbf{U}+\mathbf{D})^{-1}, \quad f \rightarrow \frac{f}{\operatorname{det}(\mathbf{C U}+\mathbf{D})},
$$


where the matrix

$$
\left(\begin{array}{ll}
\mathbf{A} & \mathbf{B} \\
\mathbf{C} & \mathbf{D}
\end{array}\right)
$$

belongs to the symplectic group $\operatorname{Sp}(4, \mathbb{R})$ (here $\mathbf{A}, \mathbf{B}, \mathbf{C}, \mathbf{D}$ are $2 \times 2$ matrices). Symmetry $(14)_{1}$ originates from linear symplectic transformations of the four-dimensional space with coordinates $u_{x}, u_{y}, x, y$, which all come from contact (Legendre-type) transformations of the first-order jet space $J^{1}\left(x, y, u, u_{x}, u_{y}\right)$, see, for example, [21] where analogous transformations of the Hessian matrix $U$ appeared in the context of integrable Hirota-type equations. Writing the corresponding linear symplectic transformation explicitly in the form

$$
\left(\begin{array}{c}
\tilde{u}_{x} \\
\tilde{u}_{y}
\end{array}\right)=\mathbf{A}\left(\begin{array}{c}
u_{x} \\
u_{y}
\end{array}\right)+\mathbf{B}\left(\begin{array}{c}
x \\
y
\end{array}\right), \quad\left(\begin{array}{c}
\tilde{x} \\
\tilde{y}
\end{array}\right)=\mathbf{C}\left(\begin{array}{l}
u_{x} \\
u_{y}
\end{array}\right)+\mathbf{D}\left(\begin{array}{l}
x \\
y
\end{array}\right),
$$

and differentiating the second set of equations, we obtain $d \tilde{x} \wedge \mathrm{d} \tilde{y}=\operatorname{det}(\mathbf{C U}+\mathbf{D}) \mathrm{d} x \wedge$ $\mathrm{d} y$. Thus,

$$
\tilde{f} \mathrm{~d} \tilde{x} \wedge \mathrm{d} \tilde{y}=\tilde{f} \operatorname{det}(\mathbf{C U}+\mathbf{D}) \mathrm{d} x \wedge \mathrm{d} y=f \mathrm{~d} x \wedge \mathrm{d} y,
$$

which explains the transformation law $(14)_{2}: \tilde{f}=f / \operatorname{det}(\mathbf{C U}+\mathbf{D})$. Similar equivalence transformations appeared in the context of first-order Lagrangians discussed in $[23,24]$. Symmetry (14) suggests a relation to Siegel modular forms (the density $f$ transforms as a genus two Siegel modular form of weight -1 ).

Furthermore, integrable Lagrangians (1) are invariant under rescalings of $f$, as well as under the addition of a 'null Lagrangian', namely transformations of the form

$$
f \rightarrow \lambda_{0} f+\lambda_{1}\left(u_{x x} u_{y y}-u_{x y}^{2}\right)+\lambda_{2} u_{x x}+\lambda_{3} u_{x y}+\lambda_{4} u_{y y}+\lambda_{5},
$$

which do not affect the Euler-Lagrange equations (here $\lambda_{i}$ are arbitrary constants). Transformations (14) and (15) generate a group of dimension $10+6=16$ which preserves the class of integrable Lagrangians (1); this group act as a point symmetry group of integrability conditions (10). Equivalence transformations will be utilised to simplify the classification results in Sect. 2.7, for instance, modulo equivalence transformations, the Lagrangian density (3) is related to $f=u_{x y}\left(u_{x x}^{2}-u_{y y}^{2}\right)$.

\subsection{Integrability conditions via generalised Rankin-Cohen brackets}

Integrability conditions (10) possess a compact formulation via higher genus RankinCohen (Eholzer-Ibukiyama) brackets for Siegel modular forms [17]. This does not come as something unexpected; indeed, the integrability conditions possess $\mathrm{Sp}(4)$ invariance (14) and, therefore, should be expressible via $\mathrm{Sp}(4)$-invariant operations. Here, we mainly follow [26,30], which specialised the general results of [17] to the 
genus two case. Let us introduce two matrix differential operators

$$
R=\left(\begin{array}{cc}
\partial_{a} & \frac{1}{2} \partial_{b} \\
\frac{1}{2} \partial_{b} & \partial_{c}
\end{array}\right), \quad S=\left(\begin{array}{cc}
\partial_{\tilde{a}} & \frac{1}{2} \partial_{\tilde{b}} \\
\frac{1}{2} \partial_{\tilde{b}} & \partial_{\tilde{c}}
\end{array}\right)
$$

(here $a, b, c$ and their tilded versions $\tilde{a}, \tilde{b}, \tilde{c}$ are the six independent variables), and define the operators $P_{0}, P_{1}, P_{2}$ via the expansion

$$
\operatorname{det}(R+\lambda S)=P_{0}+\lambda P_{1}+\lambda^{2} P_{2}
$$

Explicitly, we have

$$
P_{0}=\partial_{a} \partial_{c}-\frac{1}{4} \partial_{b}^{2}, \quad P_{1}=\partial_{a} \partial_{\tilde{c}}+\partial_{c} \partial_{\tilde{a}}-\frac{1}{2} \partial_{b} \partial_{\tilde{b}}, \quad P_{2}=\partial_{\tilde{a}} \partial_{\tilde{c}}-\frac{1}{4} \partial_{\tilde{b}}^{2}
$$

Let us also define two operators $Y_{1}, Y_{2}$ depending on the auxiliary parameters $\xi=$ $\left(\xi_{1}, \xi_{2}\right)$ by the formulae

$$
Y_{1}=\xi R \xi^{T}=\xi_{1}^{2} \partial_{a}+\xi_{1} \xi_{2} \partial_{b}+\xi_{2}^{2} \partial_{c}, \quad Y_{2}=\xi S \xi^{T}=\xi_{1}^{2} \partial_{\tilde{a}}+\xi_{1} \xi_{2} \partial_{\tilde{b}}+\xi_{2}^{2} \partial_{\tilde{c}}
$$

Finally, we introduce the $\xi$-dependent operator

$$
v=\left(\partial_{a} \partial_{\tilde{b}}-\partial_{b} \partial_{\tilde{a}}\right) \xi_{1}^{2}+2\left(\partial_{a} \partial_{\tilde{c}}-\partial_{c} \partial_{\tilde{a}}\right) \xi_{1} \xi_{2}+\left(\partial_{b} \partial_{\tilde{c}}-\partial_{c} \partial_{\tilde{b}}\right) \xi_{2}^{2}
$$

Then integrability conditions (10) can be represented in the Hirota-type bilinear form

$$
\left.\left(P_{1} Y_{1} v-2 P_{0} Y_{2} v\right)[f(a, b, c) \cdot f(\tilde{a}, \tilde{b}, \tilde{c})]\right|_{\tilde{a}=a, \tilde{b}=b, \tilde{c}=c}=0 .
$$

Here, the left-hand side is a homogeneous quartic in $\xi_{1}, \xi_{2}$, with five non-trivial components. Equating them to zero, we obtain all of the five integrability conditions (10).

Remark 5 It follows from [30], Proposition 2.3, that if $f$ transforms as in (14), that is, as a weight -1 Siegel modular form, then the left-hand side of (16) transforms as a vector-valued Siegel modular form with values in the representation $\mathrm{Sym}_{4} \otimes$ det of $\operatorname{GL}(2, \mathbb{C})$.

Remark 6 The principal symbol of the Euler-Lagrange Eq. (2) is given by a compact formula in terms of the operator $Y_{1}$ :

$$
Y_{1}^{2}[f]=f_{a a} \xi_{1}^{4}+2 f_{a b} \xi_{1}^{3} \xi_{2}+\left(2 f_{a c}+f_{b b}\right) \xi_{1}^{2} \xi_{2}^{2}+2 f_{b c} \xi_{1} \xi_{2}^{3}+f_{c c} \xi_{2}^{4}
$$

This expression transforms as a vector-valued Siegel modular form with values in the representation $\mathrm{Sym}_{4} \otimes \operatorname{det}^{-1}$. 


\subsection{Integrable Lagrangians and classical Newtonian equations}

Here, we sketch a derivation of the Lagrangian density (4). Consider a classical Newtonian equation

$$
\ddot{x}=-U_{x}
$$

where $U(x, t)$ is the potential function, $x=x(t)$, and dot denotes differentiation by $t$. This equation can be written in the canonical Hamiltonian form

$$
\dot{x}=p, \quad \dot{p}=-U_{x} .
$$

To be Liouville integrable, this Hamiltonian system should be equipped with a first integral $F(t, x, p)$ such that

$$
\frac{\mathrm{d} F}{\mathrm{~d} t} \equiv \frac{\partial F}{\partial t}+\frac{\partial F}{\partial x} \dot{x}+\frac{\partial F}{\partial p} \dot{p}=F_{t}+p F_{x}-U_{x} F_{p}=0 .
$$

First integrals $F$ polynomial in the momentum $p$ were investigated in [10] and later in [28,32]. In particular, the following cases have been studied:

$$
F=\frac{p^{3}}{3}+U p+V, \quad F=\frac{p^{4}}{4}+U p^{2}+V p+W, \quad F=\frac{p^{5}}{5}+U p^{3}+V p^{2}+W p+Q
$$

In the last (fifth-order) case, Eq. (17) implies the following quasilinear system for the coefficients:

$$
U_{t}+V_{x}=0, \quad V_{t}+W_{x}=3 U U_{x}, \quad W_{t}+Q_{x}=2 V U_{x}, \quad Q_{t}=W U_{x} .
$$

Let us introduce a potential $u$ such that $U=u_{x x}, V=-u_{x t}, W=\frac{3}{2} u_{x x}^{2}+u_{t t}$. Then the first two equations will be satisfied identically, while the last two imply

$$
Q_{x}=-2 u_{x t} u_{x x x}-3 u_{x x} u_{x x t}-u_{t t t}, \quad Q_{t}=\frac{3}{2} u_{x x}^{2} u_{x x x}+u_{t t} u_{x x x}
$$

The compatibility condition of these equations for $Q$ leads to a fourth-order PDE for $u$,

$$
u_{t t t t}+\frac{3}{2} u_{x x}^{2} u_{x x x x}+3 u_{x x} u_{x x x}^{2}+u_{t t} u_{x x x x}+2 u_{x t} u_{x x x t}+3 u_{x x} u_{x x t t}+3 u_{x t t} u_{x x x}+3 u_{x x t}^{2}=0,
$$

which is nothing but the Euler-Lagrange equation for the second-order Lagrangian

$$
S=\int\left[u_{t t}^{2}+u_{x x}^{2} u_{t t}+u_{x x} u_{x t}^{2}+\frac{1}{4} u_{x x}^{4}\right] \mathrm{d} x \mathrm{~d} t,
$$

whose density is identical to (4) up to relabelling $t \leftrightarrow y$. 


\subsection{Integrable Lagrangians and WDVV equations}

Let $F\left(t_{1}, \ldots, t_{n}\right)$ be a function of $n$ independent variables such that the symmetric matrix

$$
\eta_{i j}=\partial_{1} \partial_{i} \partial_{j} F
$$

is constant and non-degenerate (thus, $t_{1}$ is a marked variable), and the coefficients

$$
c_{j k}^{i}=\eta^{i s} \partial_{s} \partial_{j} \partial_{k} F
$$

satisfy the associativity condition $c_{i j}^{s} c_{s k}^{r}=c_{k j}^{s} c_{s i}^{r}$; here, $i, j, k \in\{1, \ldots, n\}$. These requirements impose a nonlinear system of third-order PDEs for the prepotential $F$, the so-called associativity (WDVV) equations which were discovered in the beginning of 1990s by Witten, Dijkgraaf, Verlinde and Verlinde in the context of two-dimensional topological field theory $[9,39]$. Geometry and integrability of WDVV equations has been thoroughly studied by Dubrovin, culminating in the remarkable theory of Frobenius manifolds [13]. An important ingredient of this theory is an integrable hydrodynamic hierarchy whose 'primary' part is defined by $n$ commuting Hamiltonian flows

$$
\partial_{T_{\alpha}} t_{i}=c_{\alpha k}^{i} \partial_{X} t_{k}=\partial_{X}\left(\eta^{i s} \partial_{s} \partial_{\alpha} F\right)
$$

where $T_{\alpha}$ are the higher 'times'; note that $T_{1}=X$ so that $T_{1}$-flow is trivial: $\left(t_{i}\right)_{X}=\left(t_{i}\right)_{X}$. The flows (18) are manifestly Hamiltonian with the Hamiltonian operator $\eta^{i s} \frac{\mathrm{d}}{\mathrm{d} X}$ and the Hamiltonian density $\partial_{\alpha} F$. The WDVV equations are equivalent to the requirement of commutativity of these flows.

We will need a particular case of the general construction when $n=4$ and the matrix $\eta$ is anti-diagonal, which corresponds to prepotentials

$$
F\left(t_{1}, t_{2}, t_{3}, t_{4}\right)=\frac{1}{2} t_{1}^{2} t_{4}+t_{1} t_{2} t_{3}+W\left(t_{2}, t_{3}, t_{4}\right) .
$$

The corresponding primary flows (18) take the form

$$
\begin{aligned}
& \partial_{T_{2}} t_{1}=\partial_{X}\left(\partial_{4} \partial_{2} F\right), \quad \partial_{T_{2}} t_{2}=\partial_{X}\left(\partial_{3} \partial_{2} F\right), \quad \partial_{T_{2}} t_{3}=\partial_{X}\left(\partial_{2} \partial_{2} F\right), \quad \partial_{T_{2}} t_{4}=\partial_{X}\left(\partial_{1} \partial_{2} F\right), \\
& \partial_{T_{3}} t_{1}=\partial_{X}\left(\partial_{4} \partial_{3} F\right), \quad \partial_{T_{3}} t_{2}=\partial_{X}\left(\partial_{3} \partial_{3} F\right), \quad \partial_{T_{3}} t_{3}=\partial_{X}\left(\partial_{2} \partial_{3} F\right), \quad \partial_{T_{3}} t_{4}=\partial_{X}\left(\partial_{1} \partial_{3} F\right), \\
& \partial_{T_{4}} t_{1}=\partial_{X}\left(\partial_{4} \partial_{4} F\right), \quad \partial_{T_{4}} t_{2}=\partial_{X}\left(\partial_{3} \partial_{4} F\right), \quad \partial_{T_{4}} t_{3}=\partial_{X}\left(\partial_{2} \partial_{4} F\right), \quad \partial_{T_{4}} t_{4}=\partial_{X}\left(\partial_{1} \partial_{4} F\right),
\end{aligned}
$$

which are Hamiltonian systems with the Hamiltonian densities

$$
\partial_{2} F=t_{1} t_{3}+\partial_{2} W, \quad \partial_{3} F=t_{1} t_{2}+\partial_{3} W, \quad \partial_{4} F=\frac{1}{2} t_{1}^{2}+\partial_{4} W,
$$


respectively. In compact form, Eq. (20) can be represented as

$$
\partial_{T_{\alpha}} t_{i}=\partial_{X}\left(\partial_{5-i} \partial_{\alpha} F\right), \quad i=1,2,3,4, \quad \alpha=2,3,4
$$

Setting $\left(t_{1}, t_{2}, t_{3}, t_{4}\right)=(P, B, C, A)$, we obtain

$$
F=\frac{1}{2} P^{2} A+P B C+W(B, C, A)
$$

In this case, WDVV equations reduce to the following system of four PDEs for $W$ :

$$
\begin{aligned}
W_{A A A} & =W_{A B C}^{2}+W_{A B B} W_{A C C}-W_{A A B} W_{B C C}-W_{A A C} W_{B B C}, \\
W_{A A B} & =W_{B B B} W_{A C C}-W_{A B B} W_{B C C}, \\
W_{A A C} & =W_{A B B} W_{C C C}-W_{A C C} W_{B B C}, \\
2 W_{A B C} & =W_{B B B} W_{C C C}-W_{B B C} W_{B C C} .
\end{aligned}
$$

The corresponding primary flows (20) take the form

$$
\begin{gathered}
A_{T_{2}}=C_{X}, \quad B_{T_{2}}=\left(P+W_{B C}\right)_{X}, \quad C_{T_{2}}=\left(W_{B B}\right)_{X}, P_{T_{2}}=\left(W_{A B}\right)_{X}, \\
A_{T_{3}}=B_{X}, \quad B_{T_{3}}=\left(W_{C C}\right)_{X}, \quad C_{T_{3}}=\left(P+W_{B C}\right)_{X}, \quad P_{T_{3}}=\left(W_{A C}\right)_{X}, \\
A_{T_{4}}=P_{X}, \quad B_{T_{4}}=\left(W_{A C}\right)_{X}, \quad C_{T_{4}}=\left(W_{A B}\right)_{X}, P_{T_{4}}=\left(W_{A A}\right)_{X} .
\end{gathered}
$$

Note that system (22) 2 coincides with (9) under the identification $h=W_{C}$, thus establishing a link between WDVV equations and integrable Lagrangians. This link can be summarised as follows:

- Take prepotential of type (19), set $\left(t_{2}, t_{3}, t_{4}\right)=(B, C, A)$ and define $h(A, B, C)=$ $W_{C}$.

- Reconstruct Lagrangian density $f(a, b, c)$ by applying partial Legendre transform to $h(A, B, C)$ :

$$
a=A, b=B, c=h_{C}, f=C h_{C}-h, f_{a}=-h_{A}, f_{b}=-h_{B}, f_{c}=C .
$$

Examples of calculations of this kind will be given in Sect. 2.7.5.

Remark 7 Conversely, given a Lagrangian density $f(a, b, c)$, the corresponding prepotential $W(A, B, C)$ can be reconstructed in quadratures via the formulae

$$
\begin{array}{r}
W_{A A}=-\rho_{a}, \quad W_{A B}=-\frac{1}{2} \rho_{b}, \quad W_{A C}=-f_{a}, \\
W_{B B}=-\rho_{c}, \quad W_{B C}=-f_{b}, \quad W_{C C}=c . \\
A=a, \quad B=b, \quad C=f_{c},
\end{array}
$$

where $\rho$ is defined by formulae (13), see Sect. 2.7.6. 


\subsection{Examples of integrable Lagrangians in 2D}

In this section, we present explicit examples of integrable Lagrangian densities $f$ obtained by assuming a suitable ansatz for $f$ and computing the corresponding integrability conditions (10). This gives a whole range of integrable densities expressible via polynomials, elementary functions, Jacobi theta functions and dilogarithms.

\subsubsection{Integrable Lagrangian densities of the form $f=g\left(u_{x x}, u_{y y}\right)$}

In this case, the integrability conditions lead to the only constraint $g_{a a} g_{c c}-g_{a c}^{2}=k$ where $k=$ const. Its solutions can be represented parametrically; thus, for $k=0$ (parabolic case) and $k=-1$ (hyperbolic case) we obtain the general solution in parametric form:

$$
a=p^{\prime}(w) v+q^{\prime}(w), \quad c=v, \quad f=w\left[p^{\prime}(w) v+q^{\prime}(w)\right]-[p(w) v+q(w)],
$$

and

$$
\begin{aligned}
& a=p^{\prime}(w+v)+q^{\prime}(w-v), \quad c=v \\
& \quad f=w\left[p^{\prime}(w+v)+q^{\prime}(w-v)\right]-[p(w+v)+q(w-v)]
\end{aligned}
$$

respectively; here, $p$ and $q$ are arbitrary functions, and prime denotes differentiation.

\subsubsection{Lagrangian densities for linearisable Euler-Lagrange equations}

Applying transformations from the equivalence group to quadratic densities $f$ (which generate linear Euler-Lagrange equations), we obtain Lagrangian densities whose Euler-Lagrange equations are linearisable. To state their explicit form, we introduce the variables $\left(r^{0}, r^{1}, r^{2}, r^{3}, r^{4}\right)=\left(1, a, b, c, a c-b^{2}\right)$, the minors of the Hessian matrix $\mathbf{U}$. These variables satisfy the quadratic relation

$$
r^{0} r^{4}-r^{1} r^{3}+\left(r^{2}\right)^{2}=0
$$

which defines the Plücker image of the Lagrangian Grassmannian $\Lambda$. Let $P_{i j}$ be the $5 \times 5$ symmetric matrix of this quadratic form, and let $P^{i j}$ be its inverse:

$$
P_{i j}=\left(\begin{array}{ccccc}
0 & 0 & 0 & 0 & \frac{1}{2} \\
0 & 0 & 0 & -\frac{1}{2} & 0 \\
0 & 0 & 1 & 0 & 0 \\
0 & -\frac{1}{2} & 0 & 0 & 0 \\
\frac{1}{2} & 0 & 0 & 0 & 0
\end{array}\right), \quad P^{i j}=\left(\begin{array}{ccccc}
0 & 0 & 0 & 0 & 2 \\
0 & 0 & 0 & -2 & 0 \\
0 & 0 & 1 & 0 & 0 \\
0 & -2 & 0 & 0 & 0 \\
2 & 0 & 0 & 0 & 0
\end{array}\right) .
$$

Lagrangian densities with linearisable Euler-Lagrange equations are given by the formula

$$
f=\frac{Q_{i j} r^{i} r^{j}}{S_{k} r^{k}}
$$


where the quadratic form $Q$ and the covector $S$ satisfy the following conditions:

- Covector $S$ is null with respect to the quadratic form $P$, that is, $P^{i j} S_{i} S_{j}=0$. Explicitly, $4 S_{0} S_{4}-4 S_{1} S_{3}+S_{2}^{2}=0$. (Equivalently, $S$ belongs to the dual variety $\Lambda^{*}$.)

- The dual vector $S^{i}=P^{i j} S_{j}$ lies in the kernel of the quadratic form $Q$, that is, $Q_{i j} S^{j}=0$. Equivalently, the space dual to $T_{S} \Lambda^{*}$ (tangent space to $\Lambda^{*}$ at $S$ ) lies in the kernel of $Q$.

Note that there also exist integrable densities of type (23) which do not satisfy the above conditions.

\subsubsection{Integrable Lagrangian densities of the form $f=e^{u_{x x}} g\left(u_{x y}, u_{y y}\right)$}

We will show that the generic integrable density of this form corresponds to

$$
g(b, c)=[\Delta(i c / \pi)]^{-1 / 8} \theta_{1}(b, i c / \pi)
$$

where $\Delta$ is the modular discriminant and $\theta_{1}$ is the Jacobi theta function. The details are as follows. Substituting $f=e^{a} g(b, c)$ into the integrability conditions (10), one obtains

$$
\begin{aligned}
g g_{b c c} & =3 g_{c c} g_{b}-2 g_{b c} g_{c}, \\
g g_{b b b} & =g_{b} g_{b b}+4 g_{b c} g-4 g_{b} g_{c}, \\
g g_{c c c} & =g_{c} g_{c c}+2 g_{c c} g_{b b}-2\left(g_{b c}\right)^{2}, \\
g g_{b b c} & =2 g_{b} g_{b c}-g_{c} g_{b b}+2 g g_{c c}-2\left(g_{c}\right)^{2} .
\end{aligned}
$$

This over-determined system for $g$ is in involution and can be solved as follows. First of all, equation (25) implies

$$
\left(\frac{g_{b b}}{g}\right)_{b}=\left(\frac{4 g_{c}}{g}\right)_{b},
$$

so that one can set

$$
g_{c}=\frac{1}{4}\left(g_{b b}-h g\right)
$$

where $h$ is a function of $c$ only. Using (28), both (24) and (27) reduce to

$$
g_{b b b b} g-4 g_{b} g_{b b b}+3 g_{b b}^{2}=4 h\left(g g_{b b}-g_{b}^{2}\right)-4 h^{\prime} g^{2} ;
$$

here, prime denotes differentiation by $c$. Modulo (28) and (29), equation (26) implies

$$
\begin{aligned}
& g_{b b b}^{2} g^{2}+g_{b b b}\left(4 g_{b}^{3}-6 g g_{b} g_{b b}\right)-3 g_{b}^{2} g_{b b}^{2}+4 g g_{b b}^{3} \\
& \quad=4 h\left(g_{b}^{2}-g g_{b b}\right)^{2}+8 h^{\prime} g^{2}\left(g_{b}^{2}-g g_{b b}\right)+\frac{8}{3} h^{\prime \prime} g^{4} .
\end{aligned}
$$


Note that (30) can be obtained from (29) by differentiating it with respect to $c$ and using (28), (29). Similarly, differentiating (30) with respect to $c$ we obtain the Chazy equation [6] for $h$ :

$$
h^{\prime \prime \prime}=2 h h^{\prime \prime}-3 h^{\prime 2} \text {. }
$$

Equations (29) and (30) can be simplified by the substitution $v=-(\ln g)_{b b}$, which implies

$$
v_{b b}=6 v^{2}+4 h v+4 h^{\prime}
$$

and

$$
v_{b}^{2}=4 v^{3}+4 h v^{2}+8 h^{\prime} v+\frac{8}{3} h^{\prime \prime}
$$

respectively. Since (32) follows from (33) via differentiating with respect to $b$, we end up with the following compact form of integrability conditions (24)-(27):

$$
g_{c}=\frac{1}{4}\left(g_{b b}-h g\right), \quad v=-(\ln g)_{b b}, \quad v_{b}^{2}=4 v^{3}+4 h v^{2}+8 h^{\prime} v+\frac{8}{3} h^{\prime \prime}
$$

here $h$ solves the Chazy equation (31). We recall that modulo the natural $\operatorname{SL}(2, \mathbb{R})$ symmetry [7], the Chazy equation possesses three non-equivalent solutions: $h=$ $0, h=1$ and $h=\frac{1}{2} \frac{\Delta^{\prime}}{\Delta}$ where $\Delta$ is the modular discriminant. These three solutions (which correspond to rational, trigonometric and elliptic cases of the Weierstrass $\wp$ function equation in (34)) are considered separately below. Note that both the rational and trigonometric cases lead to degenerate Lagrangians, so only the elliptic case is of interest.

Rational case $h=0$. In this case, Eq. (34) simplify to

$$
g_{c}=\frac{1}{4} g_{b b}, \quad v=-(\ln g)_{b b}, \quad v_{b}^{2}=4 v^{3}
$$

which are straightforward to solve. Modulo unessential constants the generic solution of these equations is $g=e^{2 \mu b+\mu^{2} c}(b+\mu c)$ where $\mu=$ const. The corresponding Lagrangian density $f$ takes the form

$$
f=e^{u_{x x}+2 \mu u_{x y}+\mu^{2} u_{y y}}\left(u_{x y}+\mu u_{y y}\right) .
$$

Note that the change of independent variables $x=\tilde{x}, y=\tilde{y}+\mu \tilde{x}$ brings this Lagrangian to the degenerate form $\tilde{f}=e^{u_{\tilde{x} \tilde{x}}} u_{\tilde{x} \tilde{y}}$. (The order of the corresponding Euler-Lagrange equation can be reduced by two by setting $v=u_{\tilde{x}}$.)

Trigonometric case $h=1$. In this case, Eq. (34) simplify to

$$
g_{c}=\frac{1}{4}\left(g_{b b}-g\right), \quad v=-(\ln g)_{b b}, \quad v_{b}^{2}=4 v^{3}+4 v^{2}
$$


which are also straightforward to solve. Modulo unessential constants the generic solution of these equations is $g=e^{2 \mu b+\mu^{2} c} \sinh (b+\mu c)$ where $\mu=$ const. The corresponding Lagrangian density $f$ takes the form

$$
f=e^{u_{x x}+2 \mu u_{x y}+\mu^{2} u_{y y}} \sinh \left(u_{x y}+\mu u_{y y}\right)
$$

Note that the same change of variables as in the rational case brings this Lagrangian to the degenerate form $\tilde{f}=e^{u_{\tilde{x}} \tilde{x}} \sinh u_{\tilde{x} \tilde{y}}$.

Elliptic case $h=\frac{1}{2} \frac{\Delta^{\prime}}{\Delta}$, see, for example, [36]. Here the modular discriminant $\Delta$ is given by the formula

$$
\Delta(c)=(2 \pi)^{12} q \prod_{1}^{\infty}\left(1-q^{n}\right)^{24}, \quad q=e^{2 \pi i c},
$$

recall that $h$ has the $q$-expansion

$$
h(c)=\pi i E_{2}=\pi i\left(1-24 \sum_{n=1}^{\infty} \sigma_{1}(n) q^{n}\right)
$$

where $E_{2}$ is the Eisenstein series. (Here, $\sigma_{1}(n)$ is the divisor function.) Setting $g(b, c)=[\Delta(c)]^{-1 / 8} w(b, c)$, we see that the first Eq. (34) becomes the heat equation for $w$ :

$$
w_{c}=\frac{1}{4} w_{b b}, \quad v=-(\ln w)_{b b}, \quad v_{b}^{2}=4 v^{3}+4 h v^{2}+8 h^{\prime} v+\frac{8}{3} h^{\prime \prime} .
$$

The general solution of system (35) was constructed in [5]:

$$
w(b, c)=\Delta^{1 / 8} \sigma\left(b, g_{2}, g_{3}\right) e^{b^{2} h / 6}
$$

where $\sigma$ is the Weierstrass sigma function with the invariants $g_{2}=\frac{4}{3} h^{2}-8 h^{\prime}, g_{3}=$ $-\frac{8}{27} h^{3}+\frac{8}{3} h h^{\prime}-\frac{8}{3} h^{\prime \prime}$. Note that $\Delta=\pi^{6}\left(g_{2}^{3}-27 g_{3}^{2}\right)$. Thus,

$$
g(b, c)=\sigma\left(b, g_{2}, g_{3}\right) e^{b^{2} h / 6} .
$$

Remark 8 An alternative (real-valued) representation of the general solution of system (35) in terms of the Jacobi theta function $\theta_{1}$ is as follows:

$$
w(b, c)=\theta_{1}(b, i c / \pi)=2 \sum_{n=0}^{\infty}(-1)^{n} e^{-(n+1 / 2)^{2} c} \sin [(2 n+1) b]
$$


here for $h$ in the last Eq. (35) one has to use

$$
\frac{i}{\pi} h(i c / \pi)=-1+24 \sum_{n=1}^{\infty} \sigma_{1}(n) e^{-2 n c}=-1+24\left(e^{-2 c}+3 e^{-4 c}+4 e^{-6 c}+7 e^{-8 c}+\ldots\right),
$$

which is another (real-valued) solution of the Chazy equation (the Chazy equation is invariant under the scaling symmetry $h(c) \rightarrow \lambda h(\lambda c)$. Thus,

$$
g(b, c)=[\Delta(i c / \pi)]^{-1 / 8} \theta_{1}(b, i c / \pi) .
$$

Note that the function $\Delta^{-1 / 8}(\tau) \theta_{1}(z, \tau)$ appears in the theory of weak Jacobi forms. (It is a holomorphic weak Jacobi form of weight -1 and index 1/2.) We refer to [18] for the theory of (weak) Jacobi forms.

\subsubsection{Integrable Lagrangian densities polynomial in $e^{u_{x x}}$ and $e^{u_{y y}}$}

Here, we describe integrable Lagrangian densities $f$ that are linear/quadratic in $e^{u_{x x}}$ and $e^{u_{y y}}$, the coefficients being functions of $u_{x y}$ only.

\section{Linear case:}

$$
f=p_{0}+p_{1} e^{a}+p_{2} e^{c}
$$

Substituting this ansatz into the integrability conditions (and assuming $p_{1}, p_{2}$ to be nonzero), we obtain a system of ODEs for the coefficients $p_{i}(b)$ which, modulo equivalence transformations, can be simplified to

$$
p_{1}=p_{2}=p, \quad p^{\prime \prime}=p, \quad p_{0}^{\prime \prime}=\alpha / p
$$

here $\alpha=$ const (which can be set equal to 1 if nonzero) and prime denotes differentiation by $b$. Modulo equivalence transformations, these equations possess two essentially different solutions:

$$
f=\alpha e^{-b}+\left(e^{a}+e^{c}\right) e^{b} \quad \text { and } \quad f=\alpha q(b)+\left(e^{a}+e^{c}\right) \sinh b,
$$

where the function $q(b)$ satisfies $q^{\prime \prime}=\frac{1}{\sinh b}$. This implies $q^{\prime}=\ln \frac{1-e^{b}}{1+e^{b}}$, and another integration gives

$$
q(b)=L i_{2}\left(-e^{b}\right)-L i_{2}\left(e^{b}\right)
$$

where $L i_{2}$ is the dilogarithm function: $\left(L i_{2}(x)\right)^{\prime}=-\frac{\ln (1-x)}{x}$.

\section{Quadratic case:}

$$
f=p_{0}+p_{1} e^{a}+p_{2} e^{c}+p_{3} e^{2 a}+p_{4} e^{a+c}+p_{5} e^{2 c} .
$$


Substituting this ansatz into the integrability conditions we obtain a large system of ODEs for the coefficients $p_{i}(b)$ which, modulo equivalence transformations, leads to the following integrable densities (here we only present those examples that are not reducible to the linear case by a change of variables):

$$
\begin{aligned}
& f=e^{k b+a+c}, \quad f=e^{\frac{4}{\sqrt{3}} b+a+c}+e^{\frac{2}{\sqrt{3}} b+2 c}, \\
& f=\alpha e^{-\frac{1}{\sqrt{2}} b}+\alpha e^{\frac{1}{\sqrt{2}} b+a}+e^{\frac{1}{\sqrt{2}} b+c}+e^{\frac{3}{\sqrt{2}} b+a+c}, \\
& f=p e^{2 a}+2 p^{2} e^{a+c}+p e^{2 c}, \quad p=\cosh \left(\frac{2}{\sqrt{3}} b\right) .
\end{aligned}
$$

\subsubsection{Integrable Lagrangian densities from WDVV prepotentials}

In this section, we discuss polynomial prepotentials $F$ of type (19) associated with finite Coxeter groups $W$ as given in [14], p. 107. Applying the procedure outlined at the end of Sect. 2.6, we compute the corresponding integrable Lagrangian densities $f$ which, in general, will be algebraic functions of $a, b, c$ (presented below up to appropriate scaling factors).

Group $W\left(A_{4}\right)$ :

$$
\begin{aligned}
F & =\frac{1}{2} t_{1}^{2} t_{4}+t_{1} t_{2} t_{3}+\frac{1}{2} t_{2}^{3}+\frac{1}{3} t_{3}^{4}+6 t_{2} t_{3}^{2} t_{4}+9 t_{2}^{2} t_{4}^{2}+24 t_{3}^{2} t_{4}^{3}+\frac{216}{5} t_{4}^{6} \\
f & =\left(c-48 a^{3}-12 a b\right)^{3 / 2}
\end{aligned}
$$

Swapping $t_{2}$ and $t_{3}$ (which is an obvious symmetry of WDVV equations) and following the same procedure gives a polynomial density $f$ :

$$
\begin{aligned}
F & =\frac{1}{2} t_{1}^{2} t_{4}+t_{1} t_{2} t_{3}+\frac{1}{2} t_{3}^{3}+\frac{1}{3} t_{2}^{4}+6 t_{3} t_{2}^{2} t_{4}+9 t_{3}^{2} t_{4}^{2}+24 t_{2}^{2} t_{4}^{3}+\frac{216}{5} t_{4}^{6} \\
f & =54 a^{4}-6 a^{2} c+\frac{1}{6} c^{2}-6 b^{2} a .
\end{aligned}
$$

\section{Group $W\left(B_{4}\right)$ :}

$$
\begin{aligned}
F= & \frac{1}{2} t_{1}^{2} t_{4}+t_{1} t_{2} t_{3}+t_{2}{ }^{3}+\frac{t_{2} t_{3}{ }^{3}}{3}+3 t_{2}{ }^{2} t_{3} t_{4}+\frac{t_{3}{ }^{4} t_{4}}{4}+3 t_{2} t_{3}{ }^{2} t_{4}{ }^{2}+6 t_{2}{ }^{2} t_{4}{ }^{3} \\
& +t_{3}{ }^{3} t_{4}{ }^{3}+\frac{18 t_{3}{ }^{2} t_{4}{ }^{5}}{5}+\frac{18 t_{4}{ }^{9}}{7} \\
f= & 2 a C^{3}+\left(3 a^{3}+b\right) C^{2}-3 a b^{2}
\end{aligned}
$$


where $C$ is defined by the quadratic equation $3 a C^{2}+\left(6 a^{3}+2 b\right) C+\frac{6}{5} a^{2}\left(6 a^{3}+5 b\right)=c$. Swapping $t_{2}$ and $t_{3}$ gives a polynomial density $f$ :

$$
\begin{aligned}
F= & \frac{1}{2} t_{1}^{2} t_{4}+t_{1} t_{2} t_{3}+t_{3}^{3}+\frac{t_{2}^{3} t_{3}}{3}+3 t_{2} t_{3}^{2} t_{4}+\frac{t_{2}^{4} t_{4}}{4}+3 t_{2}^{2} t_{3} t_{4}^{2}+6 t_{3}^{2} t_{4}^{3}+t_{2}^{3} t_{4}^{3} \\
& \quad+\frac{18 t_{2}^{2} t_{4}^{5}}{5}+\frac{18 t_{4}^{9}}{7} \\
f= & 12 a^{6}+12 a^{4} b-2 a^{3} c-b a c+\frac{1}{12} c^{2}-\frac{1}{3} b^{3} .
\end{aligned}
$$

Group $W\left(D_{4}\right)$ :

$$
\begin{aligned}
& F=\frac{1}{2} t_{1}^{2} t_{4}+t_{1} t_{2} t_{3}+t_{2}^{3} t_{4}+t_{3}^{3} t_{4}+6 t_{2} t_{3} t_{4}^{3}+\frac{54}{35} t_{4}^{7} \\
& f=\frac{c^{2}}{12 a}-6 b a^{3}
\end{aligned}
$$

\section{Group $W\left(F_{4}\right)$ :}

$$
\begin{aligned}
& F=\frac{1}{2} t_{1}^{2} t_{4}+t_{1} t_{2} t_{3}+\frac{t_{2}^{3} t_{4}}{18}+\frac{3 t_{3}^{4} t_{4}}{4}+\frac{t_{2} t_{3}^{2} t_{4}^{3}}{2}+\frac{t_{2}^{2} t_{4}^{5}}{60}+\frac{t_{3}^{2} t_{4}^{7}}{28}+\frac{t_{4}^{13}}{2^{4} \cdot 3^{2} \cdot 11 \cdot 13} ; \\
& f=\frac{1}{\sqrt{a}}\left(a^{7}+14 b a^{3}-14 c\right)^{3 / 2} .
\end{aligned}
$$

Swapping $t_{2}$ and $t_{3}$ gives a rational density $f$ :

$$
\begin{aligned}
& F=\frac{1}{2} t_{1}^{2} t_{4}+t_{1} t_{2} t_{3}+\frac{t_{3}^{3} t_{4}}{18}+\frac{3 t_{2}^{4} t_{4}}{4}+\frac{t_{2}^{2} t_{3} t_{4}^{3}}{2}+\frac{t_{3}^{2} t_{4}^{5}}{60}+\frac{t_{2}^{2} t_{4}^{7}}{28}+\frac{t_{4}^{13}}{2^{4} \cdot 3^{2} \cdot 11 \cdot 13} ; \\
& f=\frac{a^{9}}{600}-\frac{1}{10} c a^{4}-\frac{1}{2} b^{2} a^{3}+\frac{3 c^{2}}{2 a} .
\end{aligned}
$$

\section{Group $W\left(H_{4}\right)$ :}

$$
\begin{aligned}
F= & \frac{1}{2} t_{1}^{2} t_{4}+t_{1} t_{2} t_{3}+\frac{2 t_{2}{ }^{3} t_{4}}{3}+\frac{t_{3}{ }^{5} t_{4}}{240}+\frac{t_{2} t_{3}{ }^{3} t_{4}{ }^{3}}{18}+\frac{t_{2}{ }^{2} t_{3} t_{4}{ }^{5}}{15}+\frac{t_{3}{ }^{4} t_{4}{ }^{7}}{2^{3} \cdot 3^{3} \cdot 5} \\
& +\frac{t_{2} t_{3}{ }^{2} t_{4}{ }^{9}}{2 \cdot 3^{4} \cdot 5}+\frac{8 t_{2}{ }^{2} t_{4}{ }^{11}}{3^{4} \cdot 5^{2} \cdot 11}+\frac{t_{3}{ }^{3} t_{4}{ }^{13}}{2^{2} \cdot 3^{6} \cdot 5^{2}}+\frac{2 t_{3}{ }^{2} t_{4}{ }^{19}}{3^{8} \cdot 5^{3} \cdot 19}+\frac{32 t_{4}{ }^{31}}{3^{13} \cdot 5^{6} \cdot 29 \cdot 31}
\end{aligned}
$$

where $C$ is defined by the cubic equation $\frac{a}{12} C^{3}+\frac{a^{7}}{90} C^{2}+\frac{a^{3} b}{3} C+\frac{a^{13}}{2 \cdot 3^{5} \cdot 5^{2}} C+\frac{a^{9} b}{3^{4} \cdot 5}+$ $\frac{4 a^{19}}{3^{8} \cdot 5^{3} \cdot 19}=c$. Swapping $t_{2}$ and $t_{3}$ gives a rational density $f$ :

$F=\frac{1}{2} t_{1}^{2} t_{4}+t_{1} t_{2} t_{3}+\frac{2 t_{3}^{3} t_{4}}{3}+\frac{t_{2}^{5} t_{4}}{240}+\frac{t_{2}^{3} t_{3} t_{4}^{3}}{18}+\frac{t_{2} t_{3}^{2} t_{4}^{5}}{15}+\frac{t_{2}^{4} t_{4}^{7}}{2^{3} \cdot 3^{3} \cdot 5}$ 


$$
\begin{gathered}
+\frac{t_{2}^{2} t_{3} t_{4}^{9}}{2 \cdot 3^{4} \cdot 5}+\frac{8 t_{3}^{2} t_{4}^{11}}{3^{4} \cdot 5^{2} \cdot 11}+\frac{t_{2}^{3} t_{4}^{13}}{2^{2} \cdot 3^{6} \cdot 5^{2}}+\frac{2 t_{2}^{2} t_{4}^{19}}{3^{8} \cdot 5^{3} \cdot 19}+\frac{32 t_{4}^{31}}{3^{13} \cdot 5^{6} \cdot 29 \cdot 31} \\
f=\frac{32 a^{21}}{3^{8} \cdot 5^{4} \cdot 11^{2}}+\frac{8 b a^{15}}{3^{5} \cdot 5^{3} \cdot 11}-\frac{4 c a^{10}}{5^{2} \cdot 3^{4} \cdot 11}+\frac{2 b^{2} a^{9}}{3^{4} \cdot 5^{2}}-\frac{1}{30} b c a^{4}-\frac{1}{18} b^{3} a^{3}+\frac{c^{2}}{8 a} .
\end{gathered}
$$

Non-polynomial prepotentials (19) associated with extended affine Weyl groups can be found in [11]:

$$
\begin{aligned}
& F=\frac{1}{2} t_{1}^{2} t_{4}+t_{1} t_{2} t_{3}-\frac{1}{12} t_{2}^{2} t_{3}^{2}+\frac{1}{720} t_{2} t_{3}^{5}-\frac{1}{36288} t_{3}^{8}+2 t_{2} t_{3} e^{t_{4}}+\frac{1}{6} t_{3}^{4} e^{t_{4}}+\frac{1}{2} e^{2 t_{4}}+\frac{1}{6} \frac{t_{2}^{3}}{t_{3}} \\
& f=-\frac{C^{7}}{756}+\frac{1}{48} b C^{4}+\frac{4}{3} \mathrm{e}^{a} C^{3}-2 b \mathrm{e}^{a}+\frac{b^{3}}{2 C^{2}},
\end{aligned}
$$

where $-\frac{C^{6}}{648}+\frac{1}{36} b C^{3}+2 C^{2} \mathrm{e}^{a}-\frac{1}{6} b^{2}+\frac{b^{3}}{3 C^{3}}=c$. Swapping $t_{2}$ and $t_{3}$ gives:

$$
\begin{aligned}
F & =\frac{1}{2} t_{1}^{2} t_{4}+t_{1} t_{2} t_{3}-\frac{1}{12} t_{2}^{2} t_{3}^{2}+\frac{1}{720} t_{2}^{5} t_{3}-\frac{1}{36288} t_{2}^{8}+2 t_{2} t_{3} e^{t_{4}}+\frac{1}{6} t_{2}^{4} e^{t_{4}}+\frac{1}{2} e^{2 t_{4}}+\frac{1}{6} \frac{t_{3}^{3}}{t_{2}} \\
f & =\frac{b^{5}}{80}+\frac{1}{6} c b^{3}+\frac{1}{2} c^{2} b-2 \mathrm{e}^{a} b .
\end{aligned}
$$

Modular prepotentials [2,31] give rise to modular Lagrangian densities (as an example we took prepotential 4.2.2. from [31]):

$$
\begin{aligned}
& F=\frac{1}{2} t_{1}^{2} t_{4}+t_{1} t_{2} t_{3}-\frac{1}{4} t_{2}^{2} t_{3}^{2} \gamma\left(t_{4}\right)+t_{2}^{6} g_{4}\left(t_{4}\right)+t_{2}^{4} t_{3} g_{3}\left(t_{4}\right)+t_{3}^{3} g_{1}\left(t_{4}\right) \\
& f=\frac{1}{12 g_{1}(a)}\left[c+\frac{1}{2} b^{2} \gamma(a)\right]^{2}-g_{3}(a) b^{4}
\end{aligned}
$$

Swapping $t_{2}$ and $t_{3}$ gives:

$$
\begin{aligned}
F & =\frac{1}{2} t_{1}^{2} t_{4}+t_{1} t_{2} t_{3}-\frac{1}{4} t_{2}^{2} t_{3}^{2} \gamma\left(t_{4}\right)+t_{3}^{6} g_{4}\left(t_{4}\right)+t_{3}^{4} t_{2} g_{3}\left(t_{4}\right)+t_{2}^{3} g_{1}\left(t_{4}\right) ; \\
f & =24 C^{5} g_{4}(a)+8 b C^{3} g_{3}(a),
\end{aligned}
$$

where $C$ is defined by the algebraic equation

$$
30 C^{4} g_{4}+12 b C^{2} g_{3}-\frac{1}{2} b^{2} \gamma=c .
$$

Here $g_{3}=K g_{1}^{3}, g_{4}=\frac{K g_{1}}{30}\left(g_{1}^{\prime}-\frac{1}{2} g_{1} \gamma\right)$ where the functions of $\gamma(a)$ and $g_{1}(a)$ satisfy the ODEs

$$
\gamma^{\prime}=\frac{1}{2} \gamma^{2}-72 K g_{1}^{4}, \quad g_{1}^{\prime \prime}=2 \gamma g_{1}^{\prime}-g_{1} \gamma^{\prime}
$$


$K=$ const . The above ODE system falls within Bureau's class and its solutions are given in terms of the Schwarzian triangle functions [31].

\subsubsection{WDVV prepotentials from integrable Lagrangian densities}

In view of the correspondence between integrable Lagrangians and WDVV prepotentials

$$
F\left(t_{1}, t_{2}, t_{3}, t_{4}\right)=\frac{1}{2} t_{1}^{2} t_{4}+t_{1} t_{2} t_{3}+W\left(t_{2}, t_{3}, t_{4}\right)
$$

described in Sect. 2.6, integrable Lagrangian densities $f(a, b, c)$ constructed in this paper give rise to prepotentials some of which are apparently new. Here we list some examples (omitting details of calculations; we will only present the corresponding function $W$ ).

Example 1 The polynomial Lagrangian density from Sect. 2,

$$
f=b\left(a^{2}-c^{2}\right)
$$

gives rise to the prepotential

$$
W=\frac{1}{15} t_{4}^{5}-t_{4}^{2} t_{2} t_{3}+\frac{1}{3} t_{4} t_{2}^{4}-\frac{t_{3}^{3}}{12 t_{2}} .
$$

Example 2 Lagrangian densities from Sect. 2.7.4 (linear case): the density

$$
f=\alpha e^{-b}+\left(e^{a}+e^{c}\right) e^{b}
$$

gives rise to the prepotential

$$
W=-\alpha e^{t_{4}}-\alpha e^{-t_{2}} t_{3}-e^{t_{4}} e^{t_{2}} t_{3}-\frac{1}{2} t_{2} t_{3}^{2}+\frac{t_{3}^{2}}{2} \ln t_{3}
$$

the density

$$
f=\alpha q(b)+\left(e^{a}+e^{c}\right) \sinh b
$$

gives rise to the prepotential

$$
W=\frac{1}{8} e^{2 t_{4}}-e^{t_{4}} t_{3} \sinh t_{2}-\alpha e^{t_{4}}-\alpha q\left(t_{2}\right) t_{3}+\frac{1}{2} t_{3}^{2} \ln \frac{t_{3}}{\sinh t_{2}}-\frac{3}{4} t_{3}^{2} .
$$

Here

$$
q\left(t_{2}\right)=L i_{2}\left(-e^{t_{2}}\right)-L i_{2}\left(e^{t_{2}}\right)
$$

where $L i_{2}$ is the dilogarithm function. 
Example 3 Lagrangian densities from Sect. 2.7.4 (quadratic case): the density

$$
f=e^{k b+a+c}
$$

gives rise to the prepotential

$$
W=-\frac{1}{2} t_{4} t_{3}^{2}-\frac{k}{2} t_{2} t_{3}^{2}+\frac{t_{3}^{2}}{2} \ln t_{3}
$$

the density

$$
f=e^{\frac{4}{\sqrt{3}} b+a+c}+e^{\frac{2}{\sqrt{3}} b+2 c}
$$

gives rise to the prepotential

$$
W=\frac{t_{3}^{2}}{2} \ln t_{3}-\frac{1}{2} t_{4} t_{3}^{2}-\frac{2}{\sqrt{3}} t_{2} t_{3}^{2}-\gamma e^{\frac{2}{\sqrt{3}} t_{2}+2 t_{4}} t_{3}
$$

the density

$$
f=\alpha e^{-\frac{1}{\sqrt{2}} b}+\alpha e^{\frac{1}{\sqrt{2}} b+a}+e^{\frac{1}{\sqrt{2}} b+c}+e^{\frac{3}{\sqrt{2}} b+a+c}
$$

gives rise to the prepotential

$$
W=\frac{t_{3}^{2}}{2} \ln \frac{t_{3}}{e^{\frac{1}{\sqrt{2}} t_{2}}+e^{t_{4}+\frac{3}{\sqrt{2}} t_{2}}}-\frac{\alpha^{2}}{2} e^{t_{4}}-\alpha t_{3} \frac{1+2 e^{t_{4}+\sqrt{2} t_{2}}+e^{2 t_{4}+2 \sqrt{2} t_{2}}}{e^{\frac{1}{\sqrt{2}} t_{2}}+e^{t_{4}+\frac{3}{\sqrt{2}} t_{2}}} .
$$

Example 4 The Lagrangian density $f=e^{c} g(b, a)$ from Sect. 2.7.3 gives rise to the prepotential (recall that system (10) is invariant under the interchange $a \leftrightarrow c$; for our convenience, we choose $f=e^{c} g(b, a)$ instead of $\left.f=e^{a} g(b, c)\right)$ :

$$
W=\frac{t_{3}^{2}}{2} \ln \frac{t_{3}}{g\left(t_{2}, t_{4}\right)}
$$

Here

$$
g\left(t_{2}, t_{4}\right)=\left[\Delta\left(i t_{4} / \pi\right)\right]^{-1 / 8} \theta_{1}\left(t_{2}, i t_{4} / \pi\right)
$$

where $\Delta$ is the modular discriminant and $\theta_{1}$ is the Jacobi theta function. Note the formula $\Delta^{1 / 8}\left(i t_{4} / \pi\right)=\sqrt{2 \pi^{3}} \theta_{1}^{\prime}\left(0, i t_{4} / \pi\right)$ where prime denotes derivative by $t_{2}$. The corresponding solution of WDVV equations is related to Whitham averaged one-phase solutions of NLS/Toda equations [15], see also [1,8]. 
Example 5 The Lagrangian density $f=\sqrt{b(\alpha a+\beta b)(\alpha b+\beta c)}$ from Sect. 3.4 gives rise to the prepotential

$$
W=-\frac{\beta}{4} t_{2}\left(\alpha t_{4}+\beta t_{2}\right) \ln t_{3}-\frac{\alpha}{2 \beta} t_{2} t_{3}^{2}+\frac{\beta^{2} t_{2}^{2}}{8} \ln t_{2}+\frac{1}{8}\left(\alpha t_{4}+\beta t_{2}\right)^{2} \ln \left(\alpha t_{4}+\beta t_{2}\right) .
$$

\section{Integrable Lagrangians in 3D}

In this section, we consider second-order integrable Lagrangians of the form (5),

$$
\int f\left(u_{11}, u_{12}, u_{22}, u_{13}, u_{23}, u_{33}\right) \mathrm{d} x_{1} \mathrm{~d} x_{2} \mathrm{~d} x_{3},
$$

here $u_{i j}=u_{x_{i} x_{j}}$.

\subsection{Integrability conditions}

Let us require that all travelling wave reductions of a 3D Lagrangian density to two dimensions are integrable in the sense of Sects. 2.2 and 2.4. This gives the necessary conditions for integrability which, in our particular case, prove to be also sufficient. The computational details are as follows. Consider a travelling wave reduction of a 3D Lagrangian density $f\left(u_{11}, u_{12}, u_{22}, u_{13}, u_{23}, u_{33}\right)$ obtained by setting $u\left(x_{1}, x_{2}, x_{3}\right)=$ $v(x, y)+Q$ where $x=s_{1} x_{1}+s_{3} x_{3}, y=s_{2} x_{2}+s_{3} x_{3}, s_{i}=$ const, and $Q$ is an arbitrary homogeneous quadratic form in $x_{1}, x_{2}, x_{3}$. We have

$$
\begin{aligned}
& u_{11}=s_{1}^{2} v_{x x}+\zeta_{1}, \quad u_{12}=s_{1} s_{2} v_{x y}+\zeta_{2}, \quad u_{22}=s_{2}^{2} v_{y y}+\zeta_{3}, \\
& u_{13}=s_{1} s_{3}\left(v_{x x}+v_{x y}\right)+\zeta_{4}, \quad u_{23}=s_{2} s_{3}\left(v_{x y}+v_{y y}\right)+\zeta_{5}, \quad u_{33}=s_{3}^{2}\left(v_{x x}+2 v_{x y}+v_{y y}\right)+\zeta_{6},
\end{aligned}
$$

where $\zeta_{i}$ are the coefficients of the quadratic form $Q$. Setting $v_{x x}=a, v_{x y}=b, v_{y y}=$ $c$, we obtain the reduced 2D Lagrangian density $f$ in the form

$$
\begin{aligned}
f(a, b, c)= & f\left(u_{11}, u_{12}, u_{22}, u_{13}, u_{23}, u_{33}\right) \\
= & f\left(s_{1}^{2} a+\zeta_{1}, s_{1} s_{2} b+\zeta_{2}, s_{2}^{2} c+\zeta_{3}, s_{1} s_{3}(a+b)+\zeta_{4}, s_{2} s_{3}(b+c)\right. \\
& \left.+\zeta_{5}, s_{3}^{2}(a+2 b+c)+\zeta_{6}\right) .
\end{aligned}
$$

We have the following differentiation rules:

$$
\begin{aligned}
& \partial_{a}=s_{1}^{2} \partial_{u_{11}}+s_{1} s_{3} \partial_{u_{13}}+s_{3}^{2} \partial_{u_{33}}, \\
& \partial_{b}=s_{1} s_{2} \partial_{u_{12}}+s_{1} s_{3} \partial_{u_{13}}+s_{2} s_{3} \partial_{u_{23}}+2 s_{3}^{2} \partial_{u_{33}}, \\
& \partial_{c}=s_{2}^{2} \partial_{u_{22}}+s_{2} s_{3} \partial_{u_{23}}+s_{3}^{2} \partial_{u_{33}},
\end{aligned}
$$


etc. Substituting partial derivatives of the reduced density $f(a, b, c)$ into the $2 \mathrm{D}$ integrability conditions (10), we obtain homogeneous polynomials of degree ten in $s_{1}, s_{2}, s_{3}$ whose coefficients are expressed in terms of partial derivatives of the original 3D density $f\left(u_{i j}\right)$. Equating to zero the coefficients of these polynomials, we obtain $3 \mathrm{D}$ integrability conditions for $f$. (Note that due to the presence of arbitrary constants $\zeta_{i}$ the arguments of $f$ can be viewed as independent of $s_{1}, s_{2}, s_{3}$.) The integrability conditions can be represented in compact Hirota-type form analogous to (16):

$$
\left.\left(P_{1} Y_{1} v-2 P_{0} Y_{2} v\right)\left[f\left(u_{i j}\right) \cdot f\left(\tilde{u}_{i j}\right)\right]\right|_{\tilde{u}_{i j}=u_{i j}}=0 .
$$

Here the operators on the left-hand side of (37) are identical to that from Sect. 2.4, with the only difference that we substitute expressions (36) (and their tilded versions) for $\partial_{a}, \partial_{b}, \partial_{c}$ and $\partial_{\tilde{a}}, \partial_{\tilde{b}}, \partial_{\tilde{c}}$. Thus,

$$
\begin{gathered}
\partial_{a}=s_{1}^{2} \partial_{u_{11}}+s_{1} s_{3} \partial_{u_{13}}+s_{3}^{2} \partial_{u_{33}}, \\
\partial_{\tilde{a}}=s_{1}^{2} \partial_{\tilde{u}_{11}}+s_{1} s_{3} \partial_{\tilde{u}_{13}}+s_{3}^{2} \partial_{\tilde{u}_{33}},
\end{gathered}
$$

etc. The left-hand side of (37) is an $\mathrm{Sp}(6)$-invariant operation which transforms a function $f$ defined on the space of $3 \times 3$ symmetric matrices $u_{i j}$ into a homogeneous form of degree four in $\xi_{1}, \xi_{2}$ and degree ten in $s_{1}, s_{2}, s_{3}$.

\subsection{Equivalence group in 3D}

Let $\mathbf{U}$ be the $3 \times 3$ Hessian matrix of the function $u\left(x_{1}, x_{2}, x_{3}\right)$. Integrable Lagrangians of type (5) are invariant under $\operatorname{Sp}(6)$-symmetry

$$
\mathbf{U} \rightarrow(\mathbf{A U}+\mathbf{B})(\mathbf{C U}+\mathbf{D})^{-1}, \quad f \rightarrow \frac{f}{\operatorname{det}(\mathbf{C U}+\mathbf{D})},
$$

where the matrix

$$
\left(\begin{array}{ll}
\mathbf{A} & \mathbf{B} \\
\mathbf{C} & \mathbf{D}
\end{array}\right)
$$

belongs to the symplectic group $\operatorname{Sp}(6, \mathbb{R})$ (here $\mathbf{A}, \mathbf{B}, \mathbf{C}, \mathbf{D}$ are $3 \times 3$ matrices). Note that symmetry (38) suggests a relation to genus three Siegel modular forms. Furthermore, integrable Lagrangians (1) are invariant under rescalings of $f$, as well as under the addition of a 'null Lagrangian', namely transformations of the form

$$
f \rightarrow \lambda_{0} f+\sum \lambda_{\sigma} U_{\sigma}
$$

where $U_{\sigma}$ denote all possible minors of the Hessian matrix $\mathbf{U}$. Transformations (38) and (39) generate a group of dimension $21+15=36$ which preserves the class of integrable Lagrangians (5). 


\subsection{Integrable Lagrangians associated with the dKP hierarchy}

Here we construct three explicit second-order integrable Lagrangian densities arising in the context of the dKP hierarchy:

$$
\begin{aligned}
& f=u_{y y}^{2}-u_{x x} u_{x t}+u_{x x}^{2} u_{y y}+u_{x x} u_{x y}^{2}+\frac{1}{4} u_{x x}^{4}, \\
& f=\left(u_{x y}-u_{t t}-u_{x x} u_{x t}+\frac{1}{3} u_{x x}^{3}\right)^{3 / 2}, \\
& f=u_{x t}^{-2}\left(u_{x t} u_{y t}-u_{x x} u_{x t}^{2}\right)^{3 / 2} .
\end{aligned}
$$

These examples come from the following dKP flows.

Case 1. The fifth-order flow of the dKP hierarchy comes from the dispersionless Lax representation

$$
p_{y}=\left(\frac{p^{2}}{2}+w\right)_{x}, \quad p_{t}=\left(\frac{p^{5}}{5}+w p^{3}+v p^{2}+b p+c\right)_{x},
$$

which gives rise to the equations

$$
\begin{aligned}
& w_{y}=v_{x}, \quad b_{x}=v_{y}+3 w w_{x}, \\
& c_{x}=b_{y}+2 v w_{x}, \quad w_{t}=b w_{x}+c_{y} .
\end{aligned}
$$

Setting $w=u_{x x}, v=u_{x y}$ and $b=u_{y y}+\frac{3}{2} u_{x x}^{2}$, we obtain two equations for $c$,

$$
c_{x}=u_{y y y}+3 u_{x x} u_{x x y}+2 u_{x y} u_{x x x}, \quad c_{y}=u_{x x t}-u_{y y} u_{x x x}-\frac{3}{2} u_{x x}^{2} u_{x x x},
$$

whose compatibility condition results in the following fourth-order PDE for $u$ :

$$
\begin{aligned}
& u_{y y y y}-u_{x x x t}+3 u_{x x} u_{x x y y}+2 u_{x y} u_{x x x y}+3 u_{x x y}^{2}+3 u_{x x x} u_{x y y}+u_{y y} u_{x x x x} \\
& +\frac{3}{2} u_{x x}^{2} u_{x x x x}+3 u_{x x} u_{x x x}^{2}=0 .
\end{aligned}
$$

This is the Euler-Lagrange equation corresponding to the polynomial Lagrangian density (40).

Case 2. Another flow of the dKP hierarchy is associated with the Lax representation

$$
p_{t}=\left(\frac{p^{3}}{3}+w p+v\right)_{x}, p_{y}=\left(\frac{p^{5}}{5}+w p^{3}+v p^{2}+b p+c\right)_{x}
$$

which gives rise to the equations

$$
b_{t}=-b w_{x}+w b_{x}-2 v v_{x}+w_{y}, \quad c_{t}=w c_{x}-b v_{x}+v_{y},
$$




$$
w_{t}=-2 w w_{x}+b_{x}, \quad v_{t}=-2 v w_{x}-2 w v_{x}+c_{x} .
$$

Setting $w=u_{x x}, b=u_{x t}+u_{x x}^{2}$ and $v^{2}=u_{x y}-u_{t t}-u_{x x} u_{x t}+\frac{1}{3} u_{x x}^{3}$, we obtain two equations for $c$,

$$
c_{t}=v_{y}+u_{x x} v_{t}+2 v u_{x x} u_{x x x}+\left(u_{x x}^{2}-u_{x t}\right) v_{x}, \quad c_{x}=v_{t}+2 v u_{x x x}+2 u_{x x} v_{x},
$$

whose compatibility condition yields

$$
v_{t t}+\left(v u_{x x}\right)_{x t}=v_{x y}+\left(v\left(u_{x x}^{2}-u_{x t}\right)\right)_{x x} .
$$

This PDE is the Euler-Lagrange equation corresponding to the density (41).

Case 3. This example comes from the dispersionless Lax pair

$$
p_{t}=\left(\frac{r}{p-q}\right)_{x}, \quad p_{y}=\left(\frac{p^{3}}{3}+w p+v\right)_{x},
$$

which gives rise to the equations

$$
q_{y}=q^{2} q_{x}+q w_{x}+w q_{x}+v_{x}, \quad r_{y}=q^{2} r_{x}+2 q r q_{x}+r w_{x}+w r_{x}, \quad w_{t}=-r_{x}, \quad v_{t}=-q r_{x}-r q_{x} .
$$

Setting $w=u_{x x}, r=-u_{x t}, q^{2}=\frac{u_{y t}}{u_{x t}}-u_{x x}$, the second and the third equations will be satisfied identically, while the first and the fourth imply

$$
v_{x}=q_{y}-\left(\frac{1}{3} q^{3}+q u_{x x}\right)_{x}, \quad v_{t}=\left(u_{x t} q\right)_{x} .
$$

Their consistency condition gives

$$
q_{y t}-\left[\frac{1}{3} q\left(\frac{u_{y t}}{u_{x t}}+2 u_{x x}\right)\right]_{x t}=\left(u_{x t} q\right)_{x x} .
$$

This is the Euler-Lagrange equation corresponding to the density (42).

\subsection{D densities as travelling wave reductions of 3D densities}

Given a 3D integrable Lagrangian density $f\left(u_{x x}, u_{x y}, u_{y y}, u_{x t}, u_{y t}, u_{t t}\right)$, one can apply a travelling wave ansatz, $u(x, y, t)=u(\xi, \eta)$ where $\xi=a_{1} x+a_{2} y+a_{3} t, \eta=$ $b_{1} x+b_{2} y+b_{3} t$, to obtain an integrable 2D Lagrangian density of the form $f\left(u_{\xi \xi}, u_{\xi \eta}, u_{\eta \eta}\right)$. In fact, modulo linear transformations of $\xi$ and $\eta$ it is sufficient to assume $\xi=x+\alpha t, \eta=y+\beta t$. For instance, the two-dimensional density (4) is the stationary ( $t$-independent) reduction in the three-dimensional density (40). Similarly, applying travelling wave reduction to the $3 \mathrm{D}$ integrable density $f=\sqrt{u_{x y} u_{x t} u_{y t}}$ 
one obtains $2 \mathrm{D}$ integrable densities of the form

$$
f=\sqrt{u_{\xi \eta}\left(\alpha u_{\xi \xi}+\beta u_{\xi \eta}\right)\left(\alpha u_{\xi \eta}+\beta u_{\eta \eta}\right)} .
$$

\section{Dispersive deformations of integrable Lagrangian densities}

Some integrable Lagrangian densities $f$ possess integrable dispersive deformations $f_{\epsilon}$ of the form

$$
f_{\epsilon}=f+\epsilon f_{1}+\epsilon^{2} f_{2}+\ldots
$$

where $f$ is a second-order integrable density, $\epsilon$ is a deformation parameter and $f_{i}$ are higher-order differential expressions in $u$ of total degree $i$ (we say that second-order derivatives of $u$ have degree zero, third-order derivatives of $u$ have degree 1, etc). We require that the Euler-Lagrange equation of the deformed density $f_{\epsilon}$ is integrable (in the usual solitonic sense), so that $f$ can be seen as a dispersionless limit of $f_{\epsilon}$ when $\epsilon \rightarrow 0$. Dispersive deformations regularise the gradient catastrophe that typically occurs for dispersionless equations, and have been extensively studied by Dubrovin and his School in $1+1$ dimensions, see, for example, [12] and references therein.

A complete classification of integrable dispersive deformations of second-order Lagrangians is a challenging open problem. The three examples below come from higher-order flows of the KP hierarchy (and provide compact Lagrangian representations thereof). The corresponding dispersive deformations are therefore exact.

Example 1 The Lagrangian density (40),

$$
f=u_{y y}^{2}-u_{x x} u_{x t}+u_{x x}^{2} u_{y y}+u_{x x} u_{x y}^{2}+\frac{1}{4} u_{x x}^{4},
$$

(Section 3.3, case 1) possesses integrable dispersive deformation

$$
f_{\epsilon}=u_{y y}^{2}-u_{x x} u_{x t}+u_{x x}^{2} u_{y y}+u_{x x} u_{x y}^{2}+\frac{1}{4} u_{x x}^{4}+\frac{\epsilon^{2}}{8} u_{x x}^{2} u_{x x x x}-\frac{\epsilon^{2}}{2} u_{x x y}^{2}+\frac{\epsilon^{4}}{80} u_{x x x x}^{2} .
$$

The corresponding (dispersive) Euler-Lagrange equation has the Lax pair

$$
\epsilon \psi_{y}=\frac{\epsilon^{2}}{2} \psi_{x x}+a \psi, \quad \epsilon \psi_{t}=\frac{\epsilon^{5}}{5} \psi_{x x x x x}+\epsilon^{3} a \psi_{x x x}+\epsilon^{2} b \psi_{x x}+\epsilon c \psi_{x}+w \psi,
$$

(first and third Lax equations of the KP hierarchy) where $a=u_{x x}, b=u_{x y}+$ $\frac{3 \epsilon}{2} u_{x x x}, c=u_{y y}+\frac{3}{2} u_{x x}^{2}+\epsilon u_{x x y}+\frac{5 \epsilon^{2}}{4} u_{x x x x}$, and the variable $w$ is defined by the equations

$$
w_{x}=u_{y y y}+3 u_{x x} u_{x x y}+2 u_{x y} u_{x x x}+\frac{3 \epsilon}{2} u_{x x x}^{2}
$$




$$
\begin{gathered}
+\frac{3 \epsilon}{2} u_{x x} u_{x x x x}+\frac{\epsilon}{2} u_{x x y y}+\frac{3 \epsilon^{2}}{4} u_{x x x x y}+\frac{3 \epsilon^{3}}{8} u_{x x x x x x} \\
w_{y}=u_{x x t}-u_{y y} u_{x x x}-\frac{3}{2} u_{x x}^{2} u_{x x x}+\frac{3 \epsilon}{2}\left(u_{x x} u_{x x y}\right)_{x}+\frac{\epsilon}{2} u_{x y y y}+\frac{\epsilon^{2}}{4} u_{x x x y y} \\
-\frac{\epsilon^{2}}{4} u_{x x} u_{x x x x x}-\frac{\epsilon^{2}}{2} u_{x x x} u_{x x x x}+\frac{3 \epsilon^{3}}{8} u_{x x x x y}-\frac{\epsilon^{4}}{80} u_{x x x x x x x} .
\end{gathered}
$$

The stationary reduction of this example provides dispersive deformation of the twodimensional density (4).

Example 2 The Lagrangian density (41),

$$
f=\left(u_{x y}-u_{t t}-u_{x x} u_{x t}+\frac{1}{3} u_{x x}^{3}\right)^{3 / 2}
$$

(Sect. 3.3, case 2) possesses integrable dispersive deformation

$$
f_{\epsilon}=\left(u_{x y}-u_{t t}-u_{x x} u_{x t}+\frac{1}{3} u_{x x}^{3}+\frac{\epsilon^{2}}{12}\left(4 u_{x x} u_{x x x x}+3 u_{x x x}^{2}-4 u_{x x x t}\right)+\frac{\epsilon^{4}}{45} u_{x x x x x x}\right)^{3 / 2} .
$$

The corresponding dispersive Euler-Lagrange equation has the Lax pair

$$
\begin{aligned}
\epsilon \psi_{t}= & \frac{\epsilon^{3}}{3} \psi_{x x x}+\epsilon w \psi_{x}+v \psi, \quad \epsilon \psi_{y}=\frac{\epsilon^{5}}{5} \psi_{x x x x x} \\
& +\epsilon^{3} w \psi_{x x x}+\epsilon^{2}\left(v+\epsilon w_{x}\right) \psi_{x x}+\epsilon b \psi_{x}+c \psi
\end{aligned}
$$

where

$$
w=u_{x x}, \quad v=f_{\epsilon}^{1 / 3}+\frac{\epsilon}{2} u_{x x x}, \quad b=u_{x t}+u_{x x}^{2}+\frac{2 \epsilon^{2}}{3} u_{x x x x}+\epsilon v_{x},
$$

and the function $c$ is determined by the equations

$$
\begin{aligned}
& c_{x}=v_{t}+2\left(u_{x x} v\right)_{x}+\frac{2}{3} \epsilon^{2} v_{x x x}, \\
& c_{t}=v_{y}+\left(u_{x x} v\right)_{t}+\left(v u_{x x}^{2}-v u_{x t}-\epsilon v v_{x}-\frac{\epsilon^{2}}{3} u_{x x} v_{x x}-\frac{2 \epsilon^{2}}{3} v_{x} u_{x x x}+\frac{\epsilon^{2}}{3} c_{x x}-\frac{\epsilon^{4}}{5} v_{x x x x}\right)_{x} .
\end{aligned}
$$

Example 3 The Lagrangian density (42),

$$
f=u_{x t}^{-2}\left(u_{x t} u_{y t}-u_{x x} u_{x t}^{2}\right)^{3 / 2},
$$

(Sect. 3.3, case 3) possesses integrable dispersive deformation

$$
f_{\epsilon}=u_{x t}^{-2}\left(u_{x t} u_{y t}-u_{x x} u_{x t}^{2}+\frac{\epsilon^{2}}{4} u_{x x t}^{2}-\frac{\epsilon^{2}}{3} u_{x t} u_{x x x t}\right)^{3 / 2}
$$


The corresponding dispersive Euler-Lagrange equation comes from the Lax pair

$$
\epsilon \psi_{y}=\frac{\epsilon^{3}}{3} \psi_{x x x}+\epsilon w \psi_{x}+v \psi, \quad \epsilon^{2} \psi_{x t}=\epsilon q \psi_{t}+r \psi,
$$

where

$$
w=u_{x x}, \quad r=-u_{x t}, q=\left(\frac{f_{\epsilon}}{u_{x t}}\right)^{1 / 3}+\frac{\epsilon}{2} \frac{u_{x x t}}{u_{x t}},
$$

and the variable $v$ is defined by the equations

$$
v_{t}=\left(u_{x t} q\right)_{x}, v_{x}=q_{y}-\left(u_{x x} q+\frac{1}{3} q^{3}+\epsilon q q_{x}+\frac{\epsilon^{2}}{3} q_{x x}\right)_{x} .
$$

\section{Concluding remarks}

Here we list some problems for further study.

- Multi-dimensional Lagrangians. It would be of interest to describe multidimensional versions of second-order integrable Lagrangians. Thus, anti-self-dual four-manifolds with a parallel real spinor are described by the integrable 4D Dunajski system [16]

$$
\begin{aligned}
& a_{x t}+a_{y z}+u_{x x} a_{y y}+u_{y y} a_{x x}-2 u_{x y} a_{x y}=0, \\
& u_{x t}+u_{y z}+u_{y y} u_{x x}-u_{x y}^{2}=a,
\end{aligned}
$$

which can be written as a single fourth-order PDE for the function $u$. This PDE comes from the second-order Lagrangian

$$
\int\left(u_{x t}+u_{y z}+u_{y y} u_{x x}-u_{x y}^{2}\right)^{2} \mathrm{~d} x \mathrm{~d} y \mathrm{~d} z \mathrm{~d} t .
$$

Similarly, anti-self-dual scalar-flat four-manifolds (Flaherty-Park spaces, see [35] and references therein) are governed by the equations

$$
\begin{aligned}
& u_{x z}(\ln \Omega)_{y t}-u_{x y}(\ln \Omega)_{z t}-u_{z t}(\ln \Omega)_{x y}+u_{y t}(\ln \Omega)_{x z}=0, \\
& u_{x z} u_{y t}-u_{x y} u_{z t}=\Omega
\end{aligned}
$$

which are equivalent to a single fourth-order PDE for $u$. The corresponding Lagrangian is

$$
S=\int \Omega \ln \Omega \mathrm{d} x \mathrm{~d} y \mathrm{~d} z \mathrm{~d} t,
$$

where one has to substitute $\Omega=u_{x z} u_{y t}-u_{x y} u_{z t}$. 
- Multi-component Lagrangians. Our approach can be generalised in a straightforward way to describe 2-field integrable Lagrangians of the form

$$
\int f\left(u_{x}, u_{y}, v_{x}, v_{y}\right) \mathrm{d} x \mathrm{~d} y,
$$

as well as their 3D analogues,

$$
\int f\left(u_{x}, u_{y}, u_{t}, v_{x}, v_{y}, v_{t}\right) \mathrm{d} x \mathrm{~d} y \mathrm{~d} t .
$$

- Higher-order quasilinear PDEs. Similarly, one can classify third-order integrable PDEs of the form

$$
a_{1} u_{x x x}+a_{2} u_{x x y}+a_{3} u_{x y y}+a_{4} u_{y y y}=0
$$

where the coefficients $a_{i}$ are functions of the second-order derivatives $u_{x x}, u_{x y}, u_{y y}$ only. This problem also has a natural 3D analogue.

Acknowledgements We thank A. Basalaev, L. Bogdanov, Yu. Brezhnev, D. Guzzetti, V. Shramchenko, I. Strachan and A. Verbovetsky for useful discussions. EVF was supported by the EPSRC grant EP/N031369/1. MVP was supported by the Russian Foundation for Fundamental Research (grant 18-51-18007). We also thank the LMS for supporting a visit of MVP to Loughborough via a scheme 4 grant. LX was supported by the National Natural Science Foundation of China (grant numbers: 11501312, 11775121 and 11631007).

\section{Compliance with ethical standards}

Conflict of interests On behalf of all authors, the corresponding author states that there is no conflict of interest.

Open Access This article is licensed under a Creative Commons Attribution 4.0 International License, which permits use, sharing, adaptation, distribution and reproduction in any medium or format, as long as you give appropriate credit to the original author(s) and the source, provide a link to the Creative Commons licence, and indicate if changes were made. The images or other third party material in this article are included in the article's Creative Commons licence, unless indicated otherwise in a credit line to the material. If material is not included in the article's Creative Commons licence and your intended use is not permitted by statutory regulation or exceeds the permitted use, you will need to obtain permission directly from the copyright holder. To view a copy of this licence, visit http://creativecommons.org/licenses/by/4.0/.

\section{References}

1. Almeida, G.F.: Differential Geometry of Orbit space of Extended Affine Jacobi Group $A_{1}$. arXiv:1907.01436v2

2. Bertola, M.: Frobenius manifold structure on orbit space of Jacobi groups; Parts I and II. Differ. Geom. Appl. 13, 19-41 (2000) and 13, 213-223 (2000), and Jacobi groups, Jacobi forms and their applications, Ph.D. thesis, SISSA (1999)

3. Bialy, M., Mironov, A.E.: Integrable geodesic flows on 2-torus: formal solutions and variational principle. J. Geom. Phys. 87, 39-47 (2015)

4. Bogdanov, L.V., Konopelchenko, B.G.: Lattice and $q$-difference Darboux-Zakharov-Manakov systems via $\bar{\partial}$ dressing method. J. Phys. A 28(5), L173-L178 (1995) 
5. Bukhshtaber, V.M., Leikin, D.V., Pavlov, M.V.: Egorov hydrodynamic chains, the Chazy equation, and the group $S L(2, \mathbb{C})$. Funct. Anal. Appl. 37(4), 251-262 (2003)

6. Chazy, J.: Sur les équations différentiellles dont l'intégrale générale possède un coupure essentielle mobile. C. R. Acad. Sc. Paris, 150, 456-458 (1910)

7. Clarkson, P.A., Olver, P.J.: Symmetry and the Chazy equation. J. Differ. Equ. 124(1), 225-246 (1996)

8. Cutimanco, M., Shramchenko, V.: Explicit examples of Hurwitz Frobenius manifolds in genus one. J. Math. Phys. 61, 013501 (2020)

9. Dijkgraaf, R., Verlinde, E., Verlinde, H.: Topological strings in $d<1$. Nucl. Phys. B 352, 59-86 (1991)

10. Drach, J.: Sur l'intégration, par quadratures, de l'équation $\frac{d^{2} y}{d x^{2}}=F(x, y)$. Comptes Rendus Acad. Sci. Paris 168, 497-501 (1919)

11. Dubrovin, B., Strachan, I.A.B.: Youjin Zhang, Dafeng Zuo, Extended affine Weyl groups of BCD-type: their Frobenius manifolds and Landau-Ginzburg superpotentials. Adv. Math. 351, 897-946 (2019)

12. Dubrovin, B., Liu, S-Q., Zhang, Y.: On Hamiltonian perturbations of hyperbolic systems of conservation laws. I. Quasi-triviality of bi-Hamiltonian perturbations. Comm. Pure Appl. Math. 59, no. 4 (2006) 559-615

13. Dubrovin, B.: Geometry of 2D topological field theories. Integrable systems and quantum groups (Montecatini Terme, 1993), 120-348, Lecture Notes in Math., 1620, Fond. CIME/CIME Found. Subser. Springer, Berlin (1996)

14. Dubrovin, B.: Painlevé transcendents in two-dimensional topological field theory. The Painlevé property, pp. 287-412. CRM Ser. Math. Phys. Springer, New York (1999)

15. Dubrovin, B.A.: Hamiltonian partial differential equations and Frobenius manifolds. Russ. Math. Surveys 63(6), 999-1010 (2008)

16. Dunajski, M.: Anti-self-dual four-manifolds with a parallel real spinor. Proc. R. Soc. Lond. A 458, 1205-1222 (2002)

17. Eholzer, W., Ibukiyama, T.: Rankin-Cohen type differential operators for Siegel modular forms. Int. J. Math. 9(4), 443-463 (1998)

18. Eichler, M., Zagier, D.: The theory of Jacobi forms. Progress in Mathematics, 55. Birkhäuser Boston, Inc., Boston (1985)

19. Ferapontov, E.V.: Integration of weakly nonlinear hydrodynamic systems in Riemann invariants. Phys. Lett. A 158, 112-118 (1991)

20. Ferapontov, E.V., Khusnutdinova, K.R.: The Haantjes tensor and double waves for multi-dimensional systems of hydrodynamic type: a necessary condition for integrability Proc. R. Soc. A 462, 1197-1219 (2006)

21. Ferapontov, E.V., Hadjikos, L., Khusnutdinova, K.R.: Integrable equations of the dispersionless Hirota type and hypersurfaces in the Lagrangian Grassmannian. Int. Math. Res. Not. IMRN 3, 496-535 (2010)

22. Ferapontov, E.V., Mokhov, O.I.: On the Hamiltonian representation of the associativity equations. Algebraic aspects of integrable systems, 75-91, Progr. Nonlinear Differential Equations Appl., 26, Birkhäuser Boston, Boston, MA, 1997

23. Ferapontov, E.V., Khusnutdinova, K.R., Tsarev, S.P.: On a class of three-dimensional integrable Lagrangians. Commun. Math. Phys. 261(1), 225-243 (2006)

24. Ferapontov, E.V., Odesskii, A.V.: Integrable Lagrangians and modular forms. J. Geom. Phys. 60(6-8), 896-906 (2010)

25. Haantjes, J.: On $X_{m}$-forming sets of eigenvectors. Indagationes Mathematicae 17, 158-162 (1955)

26. Ibukiyama, T.: Vector valued Siegel modular forms of symmetric tensor weight of small degrees. Comment. Math. Univ. St. Pauli 61(1), 51-75 (2012)

27. Konopelchenko, B.G., Schief, W.K.: Three-dimensional integrable lattices in Euclidean spaces: conjugacy and orthogonality. R. Soc. Lond. Proc. Ser. A Math. Phys. Eng. Sci. 454, 3075-3104 (1998)

28. Kozlov, V.V.: Polynomial integrals of dynamical systems with one-and-a-half degrees of freedom. Math. Notes 45(3-4), 296-300 (1989)

29. Majda, A.: Compressible fluid flows and systems of conservation laws in several space variables. Appl. Math. Sci., vol 53. Springer, New York (1984)

30. Miyawaki, M.: Explicit construction of Rankin-Cohen-type differential operators for vector-valued Siegel modular forms. Kyushu J. Math. 55(2), 369-385 (2001)

31. Morrison, E.K., Strachan, I.A.B.: Polynomial modular Frobenius manifolds. Physica D: Nonlinear Phenomena 241(23-24), 2145-2155 (2012) 
32. Pavlov, M.V., Tsarev, S.P.: Classical mechanical systems with one-and-a-half degrees of freedom and Vlasov kinetic equation, Topology, geometry, integrable systems, and mathematical physics, pp. 337371. Amer. Math. Soc. Transl. Ser. 2, 234, Adv. Math. Sci., 67, Amer. Math. Soc., Providence (2014)

33. Pavlov, M.V., Vitolo, R.F.: On the bi-Hamiltonian geometry of WDVV equations. Lett. Math. Phys. 105(8), 1135-1163 (2015)

34. Pavlov, M.V., Vitolo, R.F.: Bi-Hamiltonian structure of the oriented associativity equation. J. Phys. A 52(20), 20LT01 (2019)

35. Takasaki, K.: Volume-preserving diffeomorphisms in integrable deformations of self-dual gravity. Phys. Lett. B 285(3), 187-190 (1992)

36. Takhtajan, L.A.: A simple example of modular forms as tau-functions for integrable equations. Theoret. Math. Phys. 93(2), 1308-1317 (1992)

37. Tsarev, S.P.: Poisson brackets and one-dimensional Hamiltonian systems of hydrodynamic type. Soviet Math. Dokl. 31, 488-491 (1985)

38. Tsarev, S.P.: The geometry of Hamiltonian systems of hydrodynamic type. The generalized hodograph method. Math. USSR Izvestiya 37, 397-419 (1991)

39. Witten, E.: On the structure of the topological phase of two-dimensional gravity. Nucl. Phys. B 340, 281-332 (1990)

Publisher's Note Springer Nature remains neutral with regard to jurisdictional claims in published maps and institutional affiliations. 